2011-09-13

\title{
Solutions to quasi-relativistic multi-configurative Hartree-Fock equations in quantum chemistry
}

\author{
Carlos Argáez García \\ Technological University Dublin \\ Michael Melgaard \\ Dublin Institute of Technology, michael.melgaard@dit.ie
}

Follow this and additional works at: https://arrow.tudublin.ie/scschmatart

Part of the Analysis Commons, and the Quantum Physics Commons

\section{Recommended Citation}

Argaez, C., \& Melgaard, M. (2012). Solutions to quasi-relativistic multi-configurative Hartree-Fock equations in quantum chemistry. Nonlinear Analysis: Theory, Methods \& Applications, vol. 75, no.1. doi:10.1016/j.na.2011.08.038

This Article is brought to you for free and open access by the School of Mathematics at ARROW@TU Dublin. It has been accepted for inclusion in Articles by an authorized administrator of ARROW@TU Dublin. For more information, please contact arrow.admin@tudublin.ie, aisling.coyne@tudublin.ie,gerard.connolly@tudublin.ie. Funder: SFI Stokes Award, SFI RFP grant

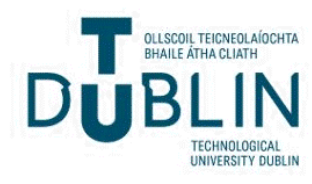




\title{
SOLUTIONS TO QUASI-RELATIVISTIC MULTI-CONFIGURATIVE HARTREE-FOCK EQUATIONS IN QUANTUM CHEMISTRY
}

\author{
C. ARGAEZ AND M. MELGAARD
}

\begin{abstract}
We establish existence of infinitely many distinct solutions to the multi-configurative Hartree-Fock type equations for $N$-electron Coulomb systems with quasi-relativistic kinetic energy $\sqrt{-\alpha^{-2} \Delta_{x_{n}}+\alpha^{-4}}-\alpha^{-2}$ for the $n^{\text {th }}$ electron. Finitely many of the solutions are interpreted as excited states of the molecule. Moreover, we prove existence of a ground state. The results are valid under the hypotheses that the total charge $Z_{\text {tot }}$ of $K$ nuclei is greater than $N-1$ and that $Z_{\text {tot }}$ is smaller than a critical charge $Z_{\mathrm{c}}$. The proofs are based on a new application of the Lions-FangGhoussoub critical point approach to nonminimal solutions on a complete analytic Hilbert-Riemann manifold.
\end{abstract}

\section{Contents}

1. Introduction

2. Preliminaries 6

3. Atomic and molecular Hamiltonians 9

4. The quasi-relativistic multi-configurative model $\quad 10$

4.1. Slater and Stiefel manifolds 11

4.2. The energy functional 12

5. Continuity properties of energy functional 14

6. The MC Hartree-Fock type equations 15

Date: December 4, 2011.

1991 Mathematics Subject Classification. Primary: 35J60; Secondary: 47J10, 58E05, 81Q10, 81V55.

Key words and phrases. Semilinear elliptic equations, multiple solutions, abstract critical point theory, Palais-Smale sequences, complete analytic RiemannHilbert manifolds.

The first author acknowledges support by grant O9-RFP-MTH23, Science Foundation Ireland (SFI). The research of the second author is supported by a Stokes Award (SFI), grant 07/SK/M1208.

THIS IS A REVISED (AND CORRECTED) VERSION OF THE ORIGINAL PAPER PUBLISHED IN NONLINEAR ANALYSIS: THEORY, METHODS, AND APPLICATIONS 75 (2012), 384-404. 
7. The quasi-relativistic Fock operator 17

8. Lower spectral bound 18

9. Convergence of Palais-Smale type sequences 19

10. Existence of a ground state 24

11. Infinitude of nonminimal solutions 25

12. Comparison with full quantum mechanical quantities $\quad 30$

References

\section{INTRODUCTION}

In this paper, we prove the existence of a solution in the form of a minimizer for a system of quasi-relativistic semilinear elliptic equations arising when one goes beyond the Hartree-Fock approximation in the modelling of an atom or molecule. Moreover, we prove the existence of infinitely many solutions to these equations, finitely many of them being interpreted as excited states for a molecule.

Within the Born-Oppenheimer approximation and expressed in Rydberg units, the quantum energy of $N$ quasi-relativistic electrons interacting with $L$ static nuclei with charges $\boldsymbol{Z}=\left(Z_{1}, \ldots, Z_{L}\right), Z_{l}>0$, is given by $\mathcal{E}\left(\boldsymbol{\Psi}_{\mathrm{e}}\right)=\left\langle\boldsymbol{\Psi}_{\mathrm{e}}, T_{N, \boldsymbol{Z}, \alpha} \boldsymbol{\Psi}_{\mathrm{e}}\right\rangle$, where $T_{N, \boldsymbol{Z}, \alpha}$ is the $N$-particle quasi-relativistic energy operator ${ }^{1}$

$$
T_{N, \boldsymbol{Z}, \alpha}=\sum_{n=1}^{N}\left\{\widetilde{T}_{0, n}+\alpha^{-1} V_{\mathrm{en}}\left(x_{n}\right)\right\}+\sum_{1 \leq m<n \leq N} V_{\mathrm{ee}}\left(x_{m}-x_{n}\right)
$$

acting on a dense subspace of the $N$-particle Hilbert space $\bigwedge_{n=1}^{N} L^{2}\left(\mathbb{R}^{3}\right)$ of antisymmetric functions. Here $\widetilde{T}_{0, n}=\sqrt{-\alpha^{-2} \Delta_{x_{n}}+\alpha^{-4}}-\alpha^{-2}$ is the quasi-relativistic kinetic energy of the $n^{\text {th }}$ electron located at $x_{n} \in \mathbb{R}^{3}$ $\left(\Delta_{x_{n}}\right.$ being the Laplacian with respect to $\left.x_{n}\right), \alpha$ is Sommerfeld's fine structure constant, $V_{\text {en }}$ is the attractive interaction between an electron and the nuclei, and $V_{\text {ee }}$ is the standard two-particle repulsive interaction between the electrons. In general, determining the infimum of $\mathcal{E}(\cdot)$ is inaccessible to direct calculation, due to the excessive dimension of the underlying Euclidean space $\mathbb{R}^{3 N}$ on which wave functions are defined. For this reason, quantum chemists have introduced ab initio approximations which provide a simplified, but still quantum mechanical description of the electronic structure about the nuclei. The idea

\footnotetext{
${ }^{1}$ Requires that $Z_{\mathrm{tot}}<Z_{\mathrm{c}}:=2 /(\alpha \pi)$. See Section 3 for details. In what follows, we ignore the spin variable but the entire contents can be trivially carried over to the spin-valued setting.
} 
of the Hartree-Fock approximation [28, 38] or, for short HF, is to replace the underlying space by a smaller space while maintaining the form of the energy $\mathcal{E}(\cdot)$. Specifically, the HF approximation consists in restricting the variational space to that of functions of the variables $\left(x_{1}, \ldots, x_{N}\right) \in \mathbb{R}^{3 N}$ that can be written as a single Slater determinant (i.e. an antisymmetrized product, see Section 2) of $N$ functions defined on $\mathbb{R}^{3}$. The corresponding Euler-Lagrange equations are known as the HF equations. For the non-relativistic setting, a review on classical results on existence of a ground state and its properties is found in Lions [31]. In the latter paper, Lions studied both minimal and nonminimal ("excited states") solutions to the equations by using critical point theory in conjunction with Morse data (see below). Lions' idea is to construct convenient min-max levels which yield the desired solutions through abstract critical point theory. For the non-relativistic HF model, Lions verifies a Palais-Smale (compactness) condition which, roughly speaking, amounts to "being away from the continuous spectrum" or, equivalently (when the so-called Morse information is taken into account), showing that certain Schrödinger operators with Coulomb type potentials have enough negative eigenvalues. Lions's method for recovering compactness from second order information was later pursued in its full generality by Fang and Ghoussoub $[15,19]$, in particular leading to streamlined versions of Lions's work on the Hartree-Fock equations. Subsequently, their work has been applied to more complicated Hartree-Fock type equations.

The multi-configurative (abbreviated MC) Hartree-Fock type equations arise as the Euler-Lagrange equations of the following variational principle $[28,38]$ : minimize $\mathcal{E}_{N}(\cdot)$ over all $N$-electron functions $\Psi:\left(\mathbb{R}^{3}\right)^{N} \rightarrow \mathbb{R}$ which are normalized and antisymmetric and, in addition, satisfy the constraint

$$
\operatorname{rank}\left(\mathcal{D}_{\boldsymbol{\Psi}_{\mathrm{e}}}\right) \leq K
$$

where $\mathcal{D}_{\boldsymbol{\Psi}_{\mathrm{e}}}$ is the one-body density operator of $\boldsymbol{\Psi}_{\mathrm{e}}$

$$
\left(\mathcal{D}_{\boldsymbol{\Psi}_{\mathrm{e}}} \phi\right)(x)=\int \mathcal{D}_{\boldsymbol{\Psi}_{\mathrm{e}}}\left(x, x^{\prime}\right) \phi\left(x^{\prime}\right) d x^{\prime}
$$

with integral kernel given by

$\mathcal{D}_{\boldsymbol{\Psi}_{\mathrm{e}}}\left(x, x^{\prime}\right)=N \int_{\mathbb{R}^{3(N-1)}} \boldsymbol{\Psi}_{\mathrm{e}}\left(x, x_{2}, \ldots, x_{n}\right) \boldsymbol{\Psi}_{\mathrm{e}}\left(x^{\prime}, x_{2}, \ldots, x_{N}\right) d x_{2} \cdots d x_{N}$.

We let $\operatorname{rank}\left(\boldsymbol{\Psi}_{\mathrm{e}}\right):=\operatorname{rank} \mathcal{D}_{\Psi_{\mathrm{e}}}$. A result by Löwdin (see Lemma 2.2) asserts that $\operatorname{rank}\left(\boldsymbol{\Psi}_{\mathrm{e}}\right) \leq K$ if and only if there exist $K L^{2}$-orthonormal one-particle functions $\left\{\phi_{n}\right\}_{n=1}^{K}$ such that $\boldsymbol{\Psi}_{\mathrm{e}}$ can be written as a linear combination of the $\left(\begin{array}{l}K \\ N\end{array}\right)$ Slater determinants (see Section 2) obtained 
by selecting any $N$ of the $\phi_{n}$ 's. When $K=N$ one obtains the abovementioned $\mathrm{HF}$ approximation. We shall use the standard formalism of MC methods (characterized by a set $\mathcal{J}$ ) which incorporates most of the those used by chemists [23, 28, 38] (See Section 6). Since an arbitrary antisymmetric wavefunction of $N$ variables can be expressed as an infinite sum of such determinants, the $\mathrm{MC}$ approximation aims at recovering more generality on the wavefunction than the $\mathrm{HF}$ approximation. The $\mathrm{MC}$ methods are thus essential to the understanding of electron correlation effects and, for this reason an enormous literature exists within Quantum Physics and Chemistry.

The aim of the present paper is threefold. Under the assumption that the total charge $Z_{\text {tot }}$ of $K$ nuclei is smaller than some critical charge $Z_{\mathrm{c}}$ and $Z_{\text {tot }}+1$ is greater than the total number of electrons $N$, we establish the following results for the quasi-relativistic multiconfigurative Hartree-Fock type equations: (1) For arbitrary $K \geq N$ and any MC method $\mathcal{J}$, a ground state exists. (2) For any $K \geq N$ and any $\mathcal{J}$, an infinitude of solutions to the equations exist. (3) For any $K \geq N$ and any $\mathcal{J}$, only a finite number of the infinitude of solutions are related to the true eigenfunctions of the full Hamiltonian $T_{N, \boldsymbol{Z}, \alpha}$. We refer to Theorems 10.1, 11.1 and 12.1 for the full statements. The results generalize those by Enstedt and Melgaard [14], who proved the existence of a ground state and an infinite number of solutions to the quasi-relativistic HF equations by implementing the above-mentioned Lions-Fang-Ghoussoub approach. We shall follow the same approach in the present paper.

We proceed to sketch the proof of Theorem 10.1. We consider the MC energy functional $\mathcal{E}_{K, N}$, see (4.12), on the Stiefel manifold $\mathcal{C}_{K, N}$, a complete metric space defined in (4.5). Since the $C^{2}$-functional $\mathcal{E}_{K, N}$ is bounded from below, we may try to find a critical point at the level $l=\inf _{\mathcal{C}_{K, N}} \mathcal{E}_{K, N}$ by determining whether the infimum is achieved. As we shall see, it is easy to find an almost critical sequence at the level $l$, that is, a sequence $\left\{\boldsymbol{h}^{(j)}\right\}$ in $\mathcal{C}_{K, N}$ satisfying

$$
\lim _{j \rightarrow \infty} \mathcal{E}_{K, N}\left(\boldsymbol{h}^{(j)}\right)=l, \quad \text { and } \quad \lim _{j \rightarrow \infty} d \mathcal{E}_{K, N}\left(\boldsymbol{h}^{(j)}\right)=0 .
$$

The hard part is to prove the existence of a converging subsequence of $\left\{\boldsymbol{h}^{(j)}\right\}$. Unfortunately, loosely speaking due to ionization, the energy functional will not satisfy a Palais-Smale condition at level $l$. To make sure that we can extract a convergent subsequence, we use second order information of $\mathcal{E}_{K, N}$.

Additional technicalities for the quasi-relativistic setting, compared to the non-relativistic, have to be resolved in order to implement these 
ideas. For instance, the Coulomb potential is not relatively compact (in the operator sense) with respect to the quasi-relativistic energy operator. In particular, compact Sobolev imbeddings are not available (for a recent survey of such problems, we refer to Bartsch-WangWillem [3]). As we shall discuss below, another complication for the functional $\mathcal{E}_{K, N}(\cdot)$ is that, contrary to the $\mathrm{HF}$ functional, it is not invariant under unitary transformations.

To address the existence of infinitely many nonminimal solutions, we invoke the direct method developed by Fang and Ghoussoub [15, 19]. Since we are looking for nonminimal (or unstable) critical points, we consider a collection $\mathrm{H}$ of compact subsets of the complete analytic Hilbert-Riemann manifold $\mathcal{C}_{N, K}$ (see Theorem 4.1) which is stable under a specific class of homotopies and then we show that $\mathcal{E}_{K, N}$ has a critical point at the level

$$
l=l_{\mathcal{E}_{K, N}, \mathrm{H}}=\inf _{\mathrm{M} \in \mathrm{H}} \max _{\boldsymbol{h} \in \mathrm{M}} \mathcal{E}_{K, N}(\boldsymbol{h}) .
$$

As we shall see, the method gives us an almost critical sequence at the level $l$, that is, a sequence $\left\{\boldsymbol{h}^{(j)}\right\}$ in $\mathcal{C}_{K, N}$ satisfying (1.3), with additional Morse information (as mentioned above) which is crucial for proving that the sequence is convergent. The method was first applied to re-derive Lions' results in the non-relativistic HF setting [15]. In fact, two different min-max levels will be introduced in order to establish Theorem 11.1, respectively Theorem 12.1.

Within the non-relativistic setting Le Bris [27] studied a variant of the case $K=N+2$ by considering the minimization problem for linear combinations of two $N$-electron Slater determinants which differ by two orbitals. The existence of a ground state was established provided $N \leq Z_{\text {tot }}$. The proof, however, does not carry over as the number of Slater determinants is increased. The general non-relativistic case, allowing many determinants under the condition $N-1<Z_{\text {tot }}$, was treated in a torce de force by Friesecke [17] who established the existence of a ground state. Lewin [29] later derived results for the nonrelativistic MC equations analogous to Lions' in the non-relativistic $\mathrm{HF}$ setting [31]. He applied the Lions-Fang-Ghoussoub approach and, for the latter, his main contribution was to devise a strategy to overcome the loss of orthogonal invariance of the functional and the possible loss of rank. We follow his scheme but several modifications are necessary.

The quasi-relativistic HF equations were investigated by Dall'Acqua et al [9], who used the Lieb-Simon method to prove the existence of a ground state for an atom provided $Z_{\text {tot }}>N-1$. In addition, regularity of the ground state away from the nucleus and pointwise exponential 
decay of the orbitals were established in [9]. For both the HF and MC orbitals, real-analyticity away from the nuclei has recently been established by Dall'Acqua et al [8]. By combining the approach of LionsFang-Ghoussoub with density operator techniques, the mathematical knowledge of the quasi-relativistic HF model was put on the same level as the non-relativistic model by Enstedt and Melgaard [14]. Other work related to our study of semilinear elliptic equations and critical point theory includes: the existence of solutions with finite Morse indices established by Dancer [10], de Figueriredo et al [11], Flores et al [16], and Tanaka [39], the existence of multiple solutions established by Cingolani-Lazzo [7] and Ghoussoub-Yuan [20], "relaxed" Palais-Smale sequences as in Lazer-Solimini [25] and Jeanjean [22], and problems on noncompact Riemannian manifolds found in Mazepa [33] and Tanaka [40].

\section{Preliminaries}

Throughout the paper we denote by $c$ and $C$ (with or without indices) various positive constants whose precise value is of no importance. Moreover, we will denote the complex conjugate of $z \in \mathbb{C}$ by $\bar{z}$.

Function spaces. For $1 \leq p \leq \infty$, let $L^{p}\left(\mathbb{R}^{3}\right)$ be the space of (equivalence classes of) complex-valued functions $\phi$ which are measurable and satisfy $\int_{\mathbb{R}^{3}}|\phi(x)|^{p} \mathrm{~d} x<\infty$ if $p<\infty$ and $\|\phi\|_{L^{\infty}\left(\mathbb{R}^{3}\right)}=\operatorname{ess} \sup |\phi|<\infty$ if $p=\infty$. The measure $\mathrm{d} x$ is the Lebesgue measure. For any $p$ the $L^{p}\left(\mathbb{R}^{3}\right)$ space is a Banach space with norm $\|\cdot\|_{L^{p}\left(\mathbb{R}^{3}\right)}=\left(\int_{\mathbb{R}^{3}} \mid \cdot\right.$ $\left.\left.\right|^{p} \mathrm{~d} x\right)^{1 / p}$. In the case $p=2, L^{2}\left(\mathbb{R}^{3}\right)$ is a complex and separable Hilbert space with scalar product $\langle\phi, \psi\rangle_{L^{2}\left(\mathbb{R}^{3}\right)}=\int_{\mathbb{R}^{3}} \phi \bar{\psi} \mathrm{d} x$ and corresponding norm $\|\phi\|_{L^{2}\left(\mathbb{R}^{3}\right)}=\langle\phi, \phi\rangle_{L^{2}\left(\mathbb{R}^{3}\right)}^{1 / 2}$. Similarly, $L^{2}\left(\mathbb{R}^{3}\right)^{N}$, the $N$ fold Cartesian product of $L^{2}\left(\mathbb{R}^{3}\right)$, is equipped with the scalar product $\langle\phi, \psi\rangle=\sum_{n=1}^{N}\left\langle\phi_{n}, \psi_{n}\right\rangle_{L^{2}\left(\mathbb{R}^{3}\right)}$. The space of infinitely differentiable complex-valued functions with compact support will be denoted $C_{0}^{\infty}\left(\mathbb{R}^{3}\right)$. The Fourier transform is given by

$$
(\mathscr{F} \psi)(\xi)=\hat{\psi}(\xi)=(2 \pi)^{-1 / 2} \int_{\mathbb{R}^{3}} e^{-i x \xi} \psi(x) \mathrm{d} x .
$$

Define

$$
\mathbf{H}^{1 / 2}\left(\mathbb{R}^{3}\right)=\left\{\phi \in L^{2}\left(\mathbb{R}^{3}\right):(1+|\xi|)^{1 / 2} \hat{\phi} \in L^{2}\left(\mathbb{R}^{3}\right)\right\},
$$

which, equipped with the scalar product

$$
\langle\phi, \psi\rangle_{\mathbf{H}^{1 / 2}\left(\mathbb{R}^{2}\right)}=\int_{\mathbb{R}^{3}}(1+|\xi|) \hat{\phi}(\xi) \overline{\hat{\psi}(\xi)} d \xi,
$$


becomes a Hilbert space; evidently, $\mathbf{H}^{1}\left(\mathbb{R}^{3}\right) \subset \mathbf{H}^{1 / 2}\left(\mathbb{R}^{3}\right)$. We have that $C_{0}^{\infty}\left(\mathbb{R}^{3}\right)$ is dense in $\mathbf{H}^{1 / 2}\left(\mathbb{R}^{3}\right)$ and the continuous embedding $\mathbf{H}^{1 / 2}\left(\mathbb{R}^{3}\right) \hookrightarrow$ $L^{3}\left(\mathbb{R}^{3}\right)$ holds; more precisely, the Sobolev inequality

$$
\|\phi\|_{L^{3}\left(\mathbb{R}^{3}\right)}^{2} \leq c_{\mathrm{sob}}\|\phi\|_{\mathbf{H}^{1 / 2}\left(\mathbb{R}^{3}\right)}^{2}
$$

is valid with $c_{\text {sob }}=2^{-1 / 2} \pi^{-2 / 3}$. Moreover, we shall use that any weakly convergent sequence in $\mathbf{H}^{1 / 2}\left(\mathbb{R}^{3}\right)$ has a pointwise convergent subsequence.

Operators. Let $T$ be a self-adjoint operator on a Hilbert space $\mathcal{H}$ with domain $\mathfrak{D}(T)$. The spectrum and resolvent set are denoted by $\sigma(T)$ and $\rho(T)$, respectively. We use standard terminology for the various parts of the spectrum; see, e.g., [12, 24]. The resolvent is $R(\zeta)=(T-\zeta)^{-1}$. The spectral family associated to $T$ is denoted by $E_{T}(\lambda), \lambda \in \mathbb{R}$. For a lower semi-bounded self-adjoint operator $T$, the counting function is defined by

$$
\operatorname{Coun}(\lambda ; T)=\operatorname{dim} \operatorname{Ran} E_{T}((-\infty, \lambda)) .
$$

We need the following abstract operator result by Lions [31, Lemma II.2].

Lemma 2.1. Let $T$ be a self-adjoint operator on a Hilbert space $\mathcal{H}$, and let $\mathcal{H}_{1}, \mathcal{H}_{2}$ be two subspaces of $\mathcal{H}$ such that $\mathcal{H}=\mathcal{H}_{1} \oplus \mathcal{H}_{2}$, $\operatorname{dim} \mathcal{H}_{1}=$ $\mathrm{h}_{1}<\infty$ and $P_{2} T P_{2} \geq 0$, where $P_{2}$ is the orthogonal projection onto $\mathcal{H}_{2}$. Then $T$ has at most $\mathrm{h}_{1}$ negative eigenvalues.

Antisymmetric wavefunctions. Let $\left\{\phi_{n}\right\}_{n=1}^{\infty}$ be an orthonormal basis of $L^{2}\left(\mathbb{R}^{3}\right)$. If we set

$$
\left(\phi_{n_{1}} \otimes \cdots \otimes \phi_{n_{N}}\right)\left(x_{1}, \ldots, x_{N}\right)=\phi_{n_{1}}\left(x_{1}\right) \cdots \phi_{n_{N}}\left(x_{N}\right),
$$

then the sequence $\left\{\phi_{n_{1}} \otimes \cdots \otimes \phi_{n_{N}}\right\}_{n_{m}=1}^{\infty}$ constitutes an orthonormal basis of $L^{2}\left(\mathbb{R}^{3 N}\right)=\otimes_{n=1}^{N} L^{2}\left(\mathbb{R}^{3}\right)$. The antisymmetrized (wedge) products $\left(\phi_{n_{1}} \wedge \cdots \wedge \phi_{n_{N}}\right)$ defined by

$$
\begin{aligned}
& \left(\phi_{n_{1}} \wedge \cdots \wedge \phi_{n_{N}}\right)\left(x_{1}, \ldots, x_{N}\right) \\
& =\frac{1}{\sqrt{N !}} \sum_{\sigma \in \mathcal{S}_{N}} \epsilon(\sigma) \phi_{n_{1}}\left(x_{\sigma(1)}\right) \cdots \phi_{n_{N}}\left(x_{\sigma(N)}\right)=\frac{1}{\sqrt{N !}} \operatorname{det}\left(\phi_{n_{l}}\left(x_{m}\right)\right)_{l, m} \\
& =\frac{1}{\sqrt{N !}}\left|\begin{array}{ccc}
\phi_{1}\left(x_{1}\right) & \cdots & \phi_{1}\left(x_{N}\right) \\
\cdot & & \cdot \\
\cdot & & \cdot \\
\cdot & & \cdot \\
\phi_{N}\left(x_{1}\right) & \cdots & \phi_{N}\left(x_{N}\right)
\end{array}\right|
\end{aligned}
$$


then give an orthonormal basis of the subspace $L_{\mathrm{a}}^{2}\left(\mathbb{R}^{3 N}\right)$ of $L^{2}\left(\mathbb{R}^{3 N}\right)$ consisting of antisymmetric functions (expressing the Pauli exclusion principle)

$$
\boldsymbol{\Psi}_{\mathrm{e}}\left(x_{1}, \ldots, x_{N}\right)=\operatorname{sign}(\sigma) \boldsymbol{\Psi}_{\mathrm{e}}\left(x_{\sigma(1)}, \ldots, x_{\sigma(N)}\right) \text { a.e. }, \quad \forall \sigma \in S_{N},
$$

where $S_{N}$ is the group of permutations of $\{1, \ldots, N\}$, with the signature of a permutation $\sigma$ being denoted by $\operatorname{sign}(\sigma)$. We write $L_{\mathrm{a}}^{2}\left(\mathbb{R}^{3 N}\right)=$ $\bigwedge_{n=1}^{N} L^{2}\left(\mathbb{R}^{3}\right)$. In the language of Quantum Chemistry, a function of the form (2.2) is called a Slater determinant. Any antisymmetric wavefunction $\Psi$ is thus an infinite linear combination of such Slater determinants

$$
\Psi_{\mathrm{e}}=\sum_{1 \leq n_{1}<\cdots<n_{N}<\infty} a_{n_{1}, \ldots, n_{N}} \phi_{n_{1}} \wedge \cdots \wedge \phi_{n_{N}}
$$

which converges in $L_{\mathrm{a}}^{2}\left(\mathbb{R}^{3 N}\right)$; in particular, the condition $\|\Psi\|_{L^{2}}=1$ is equivalent to the condition $\sum_{n_{1}<\cdots<n_{N}}\left|a_{n_{1}<\cdots<n_{N}}\right|^{2}=1$.

Fix an integer $K \geq N$ and consider the subset of $L_{\mathrm{a}}^{2}\left(\mathbb{R}^{3 N}\right)$ consisting of wave functions

$$
\Psi=\sum_{1 \leq n_{1}<\cdots<n_{N} \leq K} a_{n_{1}, \ldots, n_{N}} \phi_{n_{1}} \wedge \cdots \wedge \phi_{n_{N}},
$$

which are finite linear combinations of the $\left(\begin{array}{l}K \\ N\end{array}\right)$ Slater determinants built from a set of $K$ orthonormal functions $\left\{\phi_{n}\right\}_{n=1}^{K}$ of $L^{2}\left(\mathbb{R}^{3}\right)$. If $\left\{\phi_{n}\right\}_{n=1}^{K}$ is an orthonormal basis of the range of $\mathcal{D}_{\boldsymbol{\Psi}}$, defined in (1.2), then each $\phi_{n}$ is called an orbital of $\boldsymbol{\Psi}$. If, in addition, they are eigenfunctions of $\mathcal{D}_{\boldsymbol{\Psi}}$, the functions $\phi_{n}$ are referred to as natural orbitals, and the corresponding eigenvalues are known as the occupation numbers [38]. If $\phi=\left\{\phi_{n}\right\}_{n \in \mathbb{N}}$ is any orthonormal basis of $L^{2}\left(\mathbb{R}^{3}\right)$, then the creation operator $a^{\dagger}$ is defined as $a^{\dagger}(\phi) \phi_{n_{1}} \wedge \cdots \wedge \phi_{n_{N}}=\phi \wedge \phi_{n_{1}} \wedge \cdots \wedge \phi_{n_{N}}$ if $\phi$ is not contained in $\left\{\phi_{n_{1}}, \ldots, \phi_{n_{N}}\right\}$, and zero otherwise, and the annihilation operator $a(\phi)$ is defined as the adjoint of $a^{\dagger}(\phi)$ [38]. The following facts goes back to Löwdin [32].

\section{Lemma 2.2.}

1. Let $\left\{\phi_{n}\right\}_{n \in \mathbb{N}}$ be an orthonormal basis of $L^{2}\left(\mathbb{R}^{3}\right)$, respectively $\mathbf{H}^{1 / 2}\left(\mathbb{R}^{3}\right)$. Then $\left\{\phi_{n_{1}} \wedge \cdots \wedge \phi_{n_{N}}\right\}_{n_{1}<\cdots<n_{N}}$ is an orthonormal basis of $L_{\mathrm{a}}^{2}\left(\mathbb{R}^{3 N}\right)$, respectively $\mathbf{H}_{\mathrm{a}}^{1 / 2}\left(\mathbb{R}^{3}\right)$.

2. The set of $N$-electron wave functions of rank $K$ is nonempty if and only if

$$
K \begin{cases}=1 & N=1 \\ \geq 2, \text { even } & N=2 \\ \geq N, \neq N+1, & N \geq 3\end{cases}
$$


3. If $\boldsymbol{\Psi}$ is an $N$-electron wavefunction of rank $K$ and if $\left\{\phi_{n}\right\}_{n=1}^{K}$ is any orthonormal basis of $\operatorname{Ran} \mathcal{D}_{\boldsymbol{\Psi}}$, then $\boldsymbol{\Psi}$ is a linear combination of Slater determinants composed of the $\phi_{n}$, i.e., $\boldsymbol{\Psi}=\sum_{J} a_{J} \boldsymbol{\Phi}_{J}$ for some $a_{J} \in \mathbb{C}$, where the sum runs over multiindices $J=\left(n_{1}, \ldots, n_{N}\right) \in\{1, \ldots, K\}^{N}$, $n_{1}<\cdots<n_{N}$, and $\boldsymbol{\Phi}_{J}=\phi_{n_{1}} \wedge \cdots \wedge \phi_{n_{N}}$. Conversely, any wave function which is a linear combination of Slater determinants is of finite rank.

Assertion 1 of Lemma 2.2 merely expresses that $L_{\mathrm{a}}^{2}\left(\mathbb{R}^{3 N}\right)$ equals $\bigwedge_{n=1}^{N} L^{2}\left(\mathbb{R}^{3}\right)$. Although achievable from elementary computations using the creation and annihilation operators, $a^{\dagger}$ and $a$, for fermions and duality theory for density operators, Friesecke [17] seems to be the first who has carried out the classification of MC methods described in assertion 2. Assertion 3 is Löwdin's expansion theorem [32].

\section{Atomic and molecular Hamiltonians}

By $p$ we denote the momentum operator $-i \nabla$ on $L^{3}\left(\mathbb{R}^{3}\right)$. The operator $T_{0}=\sqrt{p^{2}+\alpha^{-2}}$ is generated by the closed, (strictly) positive form $\mathfrak{t}_{0}[\phi, \phi]=\left\langle T_{0}^{1 / 2} \phi, T_{0}^{1 / 2} \phi\right\rangle_{\mathcal{H}}$ on the form domain $\mathfrak{D}\left(\mathfrak{t}_{0}\right)=\mathbf{H}^{1 / 2}\left(\mathbb{R}^{3}\right)$. Set $S(x)=Z \alpha /|x|, Z>0, Z_{\mathrm{c}}=2 \alpha^{-1} \pi^{-1}$, and let $\widetilde{T}_{0}=T_{0}-\alpha^{-1}$. The following facts are well-known for the perturbed one-particle operator $H_{1,1, \alpha}=\widetilde{T}_{0}-S(x)[21,24]:$

Small perturbations. If $Z<\frac{\pi}{2} Z_{\mathrm{c}}$ then $S$ is $\widetilde{T}_{0}$-bounded with relative bound equal to two. If, on the other hand, $(2 \alpha)^{-1}<Z<Z_{\text {c }}$ then $S$ is $\widetilde{T}_{0}$-form bounded with relative bound less than one.

We prove the above-mentioned form-boundedness. It follows from the following inequality (first observed, it seems, by Kato [24, Paragraph VSS5.4]):

$$
\langle S \phi, \phi\rangle_{L^{2}\left(\mathbb{R}^{3}\right)} \leq\left(Z / Z_{\mathrm{C}}\right)\|\phi\|_{\mathbf{H}^{1 / 2}\left(\mathbb{R}^{3}\right)}^{2}, \quad \forall \phi \in \mathbf{H}^{1 / 2}\left(\mathbb{R}^{3}\right) .
$$

Indeed, if, for any $\psi, \phi \in \mathbf{H}^{1 / 2}\left(\mathbb{R}^{3}\right)$, we define the sesquilinear forms

$$
\begin{aligned}
\mathfrak{s}[\psi, \phi] & :=\left\langle S^{1 / 2} \psi, S^{1 / 2} \phi\right\rangle_{L^{2}\left(\mathbb{R}^{3}\right)}, \\
\mathfrak{t}_{0}[\psi, \phi] & :=\left\langle T_{0}^{1 / 2} \psi, T_{0}^{1 / 2} \phi\right\rangle_{L^{2}\left(\mathbb{R}^{3}\right)}, \\
\tilde{\mathfrak{t}}_{0}[\psi, \phi] & :=\mathfrak{t}_{0}[\psi, \phi]-\alpha^{-1}\langle\psi, \phi\rangle_{L^{2}\left(\mathbb{R}^{3}\right)},
\end{aligned}
$$

then (3.1) shows that $\mathfrak{s}$ is well-defined and also, by invoking the inequality $|-i \nabla| \leq T_{0}$, we infer that, for all $\phi \in \mathbf{H}^{1 / 2}\left(\mathbb{R}^{3}\right)$,

$$
\mathfrak{s}[\phi, \phi]<\mathfrak{t}_{0}[\phi, \phi] \text { provided } Z<Z_{\mathrm{c}} \text {. }
$$


This is the Coulomb uncertainty principle in the quasi-relativistic setting. The KLMN theorem (see, e.g., [24, Paragraph VI-1.7]) implies that there exists a unique self-adjoint operator, denoted $H_{1,1, \alpha}$, generated by the closed sesquilinear form

$$
\mathfrak{h}_{1,1, \alpha}[\psi, \phi]:=\tilde{\mathfrak{t}}_{0}[\psi, \phi]-\mathfrak{s}[\psi, \phi], \quad \psi, \phi \in \mathfrak{D}\left(\mathfrak{h}_{1,1, \alpha}\right)=\mathbf{H}^{1 / 2}\left(\mathbb{R}^{3}\right),
$$

which is bounded below by $-\alpha^{-1}$. It is well-known [21] that

$$
\begin{aligned}
\sigma\left(H_{1,1, \alpha}\right) \cap\left[-\alpha^{-1}, 0\right) & \text { is discrete } \\
\sigma\left(H_{1,1, \alpha}\right) \cap[0, \infty) & \text { is absolutely continuous }
\end{aligned}
$$

In particular,

$$
\sigma_{\text {ess }}\left(H_{1,1, \alpha}\right)=[0, \infty) .
$$

The form construction of the atomic Hamiltonian $H_{1,1, \alpha}$ can be generalized to the molecular case, describing a molecule with $N$ electrons and $K$ nuclei of charges $\boldsymbol{Z}=\left(Z_{1}, \ldots, Z_{K}\right), Z_{k}>0$, located at $R_{1}, \ldots, R_{K}$, $R_{k} \in \mathbb{R}^{3}$, if we substitute $\mathfrak{s}$ by

$$
\mathfrak{v}_{\mathrm{en}}[\psi, \phi]=\sum_{k=1}^{K}\left\langle V_{k}^{1 / 2}, \psi, V_{k}^{1 / 2} \phi\right\rangle, \quad \psi, \phi \in \mathbf{H}^{1 / 2}\left(\mathbb{R}^{3}\right),
$$

where $V_{k}$ is defined in (4.2) and by assuming that $Z_{\text {tot }}<Z_{\mathrm{c}}$.

\section{The QUASI-RElativistiC MUlTi-CONFIGURATIVE MODEL}

Within the Born-Oppenheimer approximation, the quantum energy of $N$ quasi-relativistic electrons interacting with $L$ static nuclei with charges $\boldsymbol{Z}=\left(Z_{1}, \ldots, Z_{L}\right), Z_{l}>0$, is given by

$$
\begin{aligned}
& \mathcal{E}\left(\boldsymbol{\Psi}_{\mathrm{e}}\right)=\left\langle\boldsymbol{\Psi}_{\mathrm{e}}, T_{N, \boldsymbol{Z}, \alpha} \boldsymbol{\Psi}_{\mathrm{e}}\right\rangle_{L^{2}} \\
& =\alpha^{-1} \sum_{n=1}^{N}\left\{\left\langle T_{0, n}^{\frac{1}{2}} \boldsymbol{\Psi}_{\mathrm{e}}, T_{0, n}^{\frac{1}{2}} \boldsymbol{\Psi}_{\mathrm{e}}\right\rangle_{L^{2}}-\alpha^{-1}\left\langle\boldsymbol{\Psi}_{\mathrm{e}}, \boldsymbol{\Psi}_{\mathrm{e}}\right\rangle_{L^{2}}+\left\langle V_{\mathrm{en}}\left(x_{n}\right) \boldsymbol{\Psi}_{\mathrm{e}}, \boldsymbol{\Psi}_{\mathrm{e}}\right\rangle_{L^{2}}\right\} \\
& \quad+\sum_{1 \leq m<n \leq N}\left\langle V_{\mathrm{ee}}\left(x_{m}-x_{n}\right) \boldsymbol{\Psi}_{\mathrm{e}}, \boldsymbol{\Psi}_{\mathrm{e}}\right\rangle_{L^{2}}, \quad \boldsymbol{\Psi}_{\mathrm{e}} \in \bigwedge_{n=1}^{N} \mathbf{H}^{1 / 2}\left(\mathbb{R}^{3}\right)
\end{aligned}
$$

where $x=\left(x_{1}, \ldots, x_{N}\right) \in \mathbb{R}^{3 N}, x_{n}=\left(x_{n}^{(1)}, x_{n}^{(2)}, x_{n}^{(3)}\right) \in \mathbb{R}^{3}$ is the position of the $n^{\text {th }}$ electron, $T_{0, n}=\sqrt{-\Delta_{x_{n}}+\alpha^{-2}}$ is the quasi-relativistic (one-particle) energy operator $\left(\Delta_{x_{n}}\right.$ being the square of the gradient 
$\nabla_{x_{n}}$ with respect to $x_{n}$ and $\alpha$ being Sommerfeld's fine structure constant), the potentials $V_{\text {ee }}$ and $V_{\text {en }}$ are given by

$$
V_{\text {ee }}(x)=1 /|x| \text { and } V_{\text {en }}(y)=\sum_{k=1}^{K} V_{k}(y) ; V_{k}(y)=-\frac{Z_{k} \alpha}{\left|y-R_{k}\right|}
$$

with $R_{k} \in \mathbb{R}^{3}$ being the position of the $k^{\text {th }}$ nucleus, and $T_{N, \boldsymbol{Z}, \alpha}$ is the $N$-particle quasi-relativistic energy operator given in (1.1).

The wave function $\Psi_{\mathrm{e}}:\left(\mathbb{R}^{3}\right)^{N} \rightarrow \mathbb{R}$ in (4.1) belongs to $\mathcal{H}_{\mathrm{e}}:=$ $\bigwedge^{N} \mathbf{H}^{1 / 2}\left(\mathbb{R}^{3}\right)$, i.e., the $N$-particle Hilbert space consisting of antisymmetric functions, where the Sobolev space $\mathbf{H}^{1 / 2}\left(\mathbb{R}^{3}\right)$ has been introduced in Section 2. The operator $T_{N, \boldsymbol{Z}, \alpha}$ is bounded from below and the minimum of its spectrum equals the $N$-particle ground state energy or, equivalently,

$$
E^{\mathrm{QM}}(N, \boldsymbol{Z}, \alpha)=\inf \left\{\mathcal{E}\left(\boldsymbol{\Psi}_{\mathrm{e}}\right): \boldsymbol{\Psi}_{\mathrm{e}} \in \mathcal{H}_{\mathrm{e}},\left\|\boldsymbol{\Psi}_{\mathrm{e}}\right\|_{L^{2}\left(\mathbb{R}^{3 N}\right)}=1\right\} .
$$

The bottom of the essential spectrum, $\Sigma:=\inf \sigma_{\mathrm{ess}}\left(T_{N, \boldsymbol{Z}, \alpha}\right)$, is described by the HVZ theorem, see Lewis et al [26], and the nuclei are capable of binding the $N$ electrons in their vicinity provided $E^{\mathrm{QM}}(N, \boldsymbol{Z}, \alpha)$ $<\Sigma$. For $Z_{\text {tot }} \geq N$, Zhislin and Vugalter [41] have shown that there are infinitely many eigenvalues below $\Sigma$; hence we have that

$$
\sigma\left(T_{N, \boldsymbol{Z}, \alpha}\right)=\left\{E^{\mathrm{QM}}(N, \boldsymbol{Z}, \alpha)=\nu_{1} \leq \nu_{2} \leq \cdots \nu_{i} \leq \cdots\right\} \cup[\Sigma, \infty),
$$

where $\left\{\nu_{i}\right\}_{i \geq 2}$ are eigenvalues strictly less than $\Sigma$. Their eigenfunctions are called excited states.

4.1. Slater and Stiefel manifolds. To determine $E^{\mathrm{QM}}(N, \boldsymbol{Z}, \alpha)$ directly turns out to be too difficult, even for small $N$. One of the classical approximation methods for determining $E^{\mathrm{QM}}(N, \boldsymbol{Z}, \alpha)$ is the $H F$ theory, introduced by Hartree and improved by Fock and Slater in the late 1920s (see, e.g., [38, 28]), which consists of restricting attention to simple wedge products $\Psi_{\mathrm{e}} \in \mathcal{S}_{N}$, where the Slater manifold is given by

$$
\mathcal{S}_{N}=\left\{\Psi_{\mathrm{e}} \in \mathcal{H}_{\mathrm{e}}: \exists \boldsymbol{\Phi} \text { s.t. } \Psi_{\mathrm{e}}=\frac{1}{\sqrt{N !}} \operatorname{det}\left(\phi_{n}\left(x_{m}\right)\right)\right\}
$$

and the (infinite-dimensional) Stiefel manifold $\mathcal{C}_{N}$ is given by $\mathcal{C}_{N}=\left\{\boldsymbol{\Phi}=\left\{\phi_{n}\right\}_{n=1}^{N}, \phi_{n} \in \mathbf{H}^{1 / 2}\left(\mathbb{R}^{3}\right),\left\langle\phi_{m}, \phi_{n}\right\rangle_{L^{2}}=\delta_{m n}, 1 \leq m, n \leq N\right\}$.

In the variational MC method of rank $K$ the set of admissible wavefunctions is restricted to those which can be written as a linear combination 
of Slater determinants, as in (2.3), constructed from $K L^{2}$-orthonormal functions $\phi_{1}, \ldots, \phi_{K}$. Let $\mathcal{H}_{N}^{K}=\mathbb{R}^{\left(\begin{array}{l}K \\ N\end{array}\right)} \times\left(\mathbf{H}^{1 / 2}\left(\mathbb{R}^{3}\right)\right)^{K}$ and define $^{2}$

$$
\mathcal{C}_{K, N}=\left\{(a, \boldsymbol{\Phi}) \in \mathcal{H}_{N}^{K}: \sum_{n_{1}<\cdots<n_{N}}\left|a_{n_{1}, \ldots, n_{N}}\right|^{2}=1, \int_{\mathbb{R}^{3}} \phi_{m} \phi_{n}=\delta_{m n}\right\},
$$

where

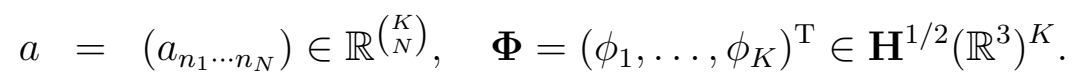

To ensure that the MC energy functional, to be defined below is welldefined, we emphasize that $\mathbf{H}^{1 / 2}\left(\mathbb{R}^{3}\right)$ regularity are imposed on the elements of $\Phi$. When no ambiguity is possible, we shall use the notation

$$
\boldsymbol{\Psi}_{\mathrm{e}}=\sum_{J \subset\{1, \ldots, K\},|J|=N} a_{J} \boldsymbol{\Phi}_{J}
$$

where $a_{J}=\left(a_{n_{1} \ldots n_{N}}\right)$ and $\boldsymbol{\Phi}_{J}=\phi_{n_{1}} \wedge \cdots \wedge \phi_{n_{N}}$ for $J=\left\{n_{1}<\cdots<n_{N}\right\}$.

Chiumiento and Melgaard [6] have proved the following result, which is needed in order to implement the Lions-Fang-Ghoussoub approach.

Theorem 4.1. The variational space $\mathcal{C}_{K, N}$ is a complete, analytic Hilbert-Riemann manifold.

4.2. The energy functional. The quasi-relativistic multi-configurative energy functional is defined by the formula

$$
\begin{gathered}
\mathcal{E}\left(\boldsymbol{\Psi}_{(a, \boldsymbol{\Phi})}\right)=\left\langle\boldsymbol{\Psi}_{(a, \boldsymbol{\Phi})}, T_{N, \boldsymbol{Z}, \alpha} \boldsymbol{\Psi}_{(a, \boldsymbol{\Phi})}\right\rangle \\
\boldsymbol{\Psi}_{(a, \boldsymbol{\Phi})}=\sum_{J} a_{J} \boldsymbol{\Phi}_{J}=\sum_{1 \leq n_{1}<\cdots<n_{N}<\infty} a_{n_{1}, \ldots, n_{N}} \phi_{n_{1}} \wedge \cdots \wedge \phi_{n_{N}} .
\end{gathered}
$$

Definition 4.2. Let $\boldsymbol{Z}=\left(Z_{1}, \ldots, Z_{L}\right), Z_{l}>0, l=1, \ldots, L$, and let $N$ be a nonnegative integer. The $N$-particle quasi-relativistic MC ground state energy is

$$
\begin{aligned}
E_{K, N}=E_{K, N}(\boldsymbol{Z}, \alpha)= & \\
=\inf \left\{\mathcal{E}\left(\boldsymbol{\Psi}_{\mathrm{e}}\right): \boldsymbol{\Psi}_{\mathrm{e}}\right. & \in \mathbf{H}_{\mathrm{a}}^{1 / 2}\left(\mathbb{R}^{3 N}\right), \\
& \left.\left\|\boldsymbol{\Psi}_{\mathrm{e}}\right\|_{L^{2}\left(\mathbb{R}^{3 N}\right)}=1, \operatorname{rank}\left(\boldsymbol{\Psi}_{\mathrm{e}}\right) \leq K\right\} .
\end{aligned}
$$

If a minimizer exists, i.e., there exists some $\boldsymbol{\Psi}_{\mathrm{e}}$ such that

$$
\mathcal{E}\left(\boldsymbol{\Psi}_{\mathrm{e}}\right)=E_{K, N}
$$

then it is said that the molecule has a quasi-relativistic MC ground state described by $\boldsymbol{\Psi}_{\mathrm{e}}$.

\footnotetext{
${ }^{2}$ The $a_{n_{1} \ldots n_{N}}$ are arranged in a column vector $a$ using, e.g., the lexicographical order.
} 
Since any wavefunction $\boldsymbol{\Psi}_{\mathrm{e}}$ can be expressed as a (infinite) linear combination of Slater determinants (Lemma 2.2), one has $\lim _{K \rightarrow \infty} E_{N, K}=$ $E^{\mathrm{QM}}(N, \boldsymbol{Z}, \alpha)$. Moreover, it is convenient to write

$$
\Psi=\sum_{n_{1}, \ldots, n_{N}} b_{n_{1}, \ldots, n_{N}} \phi_{n_{1}} \wedge \cdots \wedge \phi_{n_{N}} .
$$

where $^{3}$

$$
b_{n_{1} \cdots n_{N}}=\left\{\begin{array}{lc}
0 & \text { if }
\end{array} \quad \#\left\{n_{1} \cdots n_{N}\right\}<N,\right.
$$

Expressed in terms of $b_{J}$ and $\phi_{n}$ the functional $\mathcal{E}$ equals

$$
\begin{aligned}
& \mathcal{E}(\boldsymbol{\Psi})=N \sum_{1 \leq k_{2} \ldots \leq k_{N} \leq K}\left\{\tilde{\mathfrak{t}}_{0}\left[\sum_{n=1}^{K} b_{n, k_{2}, \ldots, k_{N}} \phi_{n}, \sum_{n=1}^{K} b_{n, k_{2}, \ldots, k_{N}} \phi_{n}\right]\right. \\
& \left.+\mathfrak{v}_{\mathrm{en}}\left[\sum_{n=1}^{K} b_{n, k_{2}, \ldots, k_{N}} \phi_{n}, \sum_{n=1}^{K} b_{n, k_{2}, \ldots, k_{N}} \phi_{n}\right]\right\} \\
& +\frac{\tilde{N}}{2} \sum_{1 \leq k_{3} \ldots \leq k_{N} \leq K} \int_{\mathbb{R}^{3}} \int_{\mathbb{R}^{3}} \frac{\left(\sum_{1 \leq m, n \leq K} b_{m, n, k_{3}, \ldots, k_{N}} \phi_{m}(x) \phi_{n}(y)\right)^{2}}{|x-y|} d x d y .
\end{aligned}
$$

where $\widetilde{N}=N(N-1)$. Let $\boldsymbol{\phi}$ be as in (4.5) and let $\langle\boldsymbol{\phi}, \widetilde{\boldsymbol{\phi}}\rangle_{L^{2}\left(\mathbb{R}^{3}\right)^{K}}=$ $\sum_{n=1}^{N} \int_{\mathbb{R}^{3}} \phi_{m} \tilde{\phi}_{n} d x$. By introducing the $K \times K$ matrices $\boldsymbol{D}$ and $\boldsymbol{E}_{\phi}$ given by

$$
\begin{aligned}
D_{m, n}= & N \sum_{k_{2}, \ldots, k_{N}} b_{m, k_{2}, \ldots, k_{N}} b_{n, k_{2}, \ldots, k_{N}} \\
\left(\boldsymbol{E}_{\phi}\right)_{m, n}(x)= & \frac{\widetilde{N}}{2} \sum_{k_{3}, \ldots, k_{N}} \sum_{i, j} b_{m, i, k_{3}, \ldots, k_{N}} b_{n, j, k_{3}, \ldots, k_{N}} \\
& \times\left(\phi_{i} \phi_{j} * \frac{1}{|x|}\right)(x),
\end{aligned}
$$

we can re-express the functional in (4.8) as follows, understood in the sense of quadratic forms as above,

$$
\mathcal{E}(\boldsymbol{\Psi})=\left\langle\left(\left(\widetilde{T}_{0}+V_{\mathrm{en}}\right) \boldsymbol{D}+\boldsymbol{E}_{\boldsymbol{\phi}}\right) \boldsymbol{\phi}, \boldsymbol{\phi}\right\rangle_{L^{2}\left(\mathbb{R}^{3}\right)^{K}},
$$

\footnotetext{
${ }^{3} \sigma$ is the permutation of $\{1, \ldots, N\}$ such that $n_{\sigma(1)}<n_{\sigma(2)}<\cdots n_{\sigma(N)}$.
} 
or, alternatively, for elements $(a, \phi)$ belonging to the Stiefel manifold $\mathcal{C}_{K, N}$ defined in (4.5),

$$
\mathcal{E}_{K, N}(a, \phi):=\left\langle\left(\left(\widetilde{T}_{0}+V_{\mathrm{en}}\right) \boldsymbol{D}+\boldsymbol{E}_{\boldsymbol{\phi}}\right) \boldsymbol{\phi}, \boldsymbol{\phi}\right\rangle_{\left.L^{2}\left(\mathbb{R}^{3}\right)\right)^{K}}, \quad(a, \boldsymbol{\phi}) \in \mathcal{C}_{K, N}
$$

On $\mathcal{C}_{K, N}$ the first derivative, respectively the second derivative, of $\mathcal{E}_{K, N}$ with respect to $\boldsymbol{\Phi}$ will be denoted by $d_{\boldsymbol{\Phi}} \mathcal{E}_{K, N}$, respectively $d_{\boldsymbol{\Phi}}^{2} \mathcal{E}_{K, N}$. By standard arguments (see, e.g., [31, 29, 13]) we obtain the following result on the regularity and orthogonal invariance of the functional $\mathcal{E}_{K, N}(\cdot)$.

\section{Lemma 4.3.}

1. Regularity. The functional $\mathcal{E}_{N}(\cdot)$, respectively $\mathcal{E}_{K, N}(\cdot)$, belongs to $C^{2}\left(\mathbf{H}^{1 / 2}\left(\mathbb{R}^{3}\right)^{K}, \mathbb{R}\right)$, respectively $C^{2}\left(\mathcal{C}_{K, N}, \mathbb{R}\right)$.

2. Orthogonal invariance. If $(\tilde{a}, \widetilde{\boldsymbol{\phi}})=\boldsymbol{U} \cdot(a, \boldsymbol{\phi})=(a, \boldsymbol{U} \boldsymbol{\phi})$ for $\boldsymbol{U} \in$ $\mathcal{O}_{K}(\mathbb{R})$ (the set of $K \times K$ orthogonal matrices) then $\widetilde{\boldsymbol{D}}=\boldsymbol{U} \boldsymbol{D} \boldsymbol{U}^{\mathrm{T}}$, $\widetilde{\boldsymbol{E}}_{\widetilde{\boldsymbol{\phi}}}=\boldsymbol{U} \boldsymbol{E}_{\boldsymbol{\phi}} \boldsymbol{U}^{\mathrm{T}}$ and $\mathcal{E}_{K, N}$ is invariant under the group action of $\mathcal{O}_{K}(\mathbb{R})$ on $\mathcal{C}_{K, N}$.

\section{Continuity PRoperties of EnERGy FUnCtional}

Lemma 5.1. The functional $\mathcal{E}_{K, N}$, defined in (4.11), is continuous on $\mathcal{C}_{K, N}$ and it is weakly lower semicontinuous on $\mathcal{H}_{N}^{K}$.

Proof. The continuity of $\mathcal{E}_{K, N}$ on $\mathcal{C}_{K, N}$ is easily shown. To establish weakly lower semicontinuity, we recall that $\phi_{i} \rightarrow \phi$ in $\mathbf{H}^{1 / 2}\left(\mathbb{R}^{3}\right)$ implies that (see, e.g., [9])

$$
\liminf _{i \rightarrow \infty}\left(\tilde{\mathfrak{t}}_{0}+\mathfrak{v}_{\mathrm{en}}\right)\left[\phi_{i}, \phi_{i}\right] \geq\left(\tilde{\mathfrak{t}}_{0}+\mathfrak{v}_{\mathrm{en}}\right)[\phi, \phi]
$$

Suppose that a sequence $\left(a^{(j)}, \phi^{(j)}\right) \in \mathcal{H}_{N}^{K}$ satisfies $a^{(j)} \rightarrow a$ and $\boldsymbol{\phi}^{(j)} \rightarrow \boldsymbol{\phi}$ weakly in $\left(\mathbf{H}^{1 / 2}\left(\mathbb{R}^{3}\right)\right)^{K}$. Then, after extracting a subsequence if necessary, we may assume that $\phi^{(j)} \rightarrow \boldsymbol{\phi}$ strongly in $\left(L_{\text {loc }}^{2}\left(\mathbb{R}^{3}\right)\right)^{K}$ (and a.e.) and, furthermore, $\mathcal{E}_{K, N}\left(a^{(j)}, \phi^{(j)}\right) \rightarrow E$, where we have defined $E:=\liminf _{j \rightarrow \infty} \mathcal{E}_{K, N}\left(a^{(j)}, \phi^{(j)}\right)$. Now, for all $k_{2}, \ldots, k_{N}$ we have that

$$
\sum_{n=1}^{K} b_{n, k_{2}, \ldots, k_{N}}^{(j)} \phi_{n}^{(j)} \rightarrow \sum_{n=1}^{K} b_{n, k_{2}, \ldots, k_{N}} \phi_{n}
$$


weakly in $\mathbf{H}^{1 / 2}\left(\mathbb{R}^{3}\right)$ and strongly in $L_{\text {loc }}^{2}\left(\mathbb{R}^{3}\right)$ and, therefore, the property (5.1) implies that

$$
\begin{gathered}
\liminf _{j \rightarrow \infty}\left(\tilde{\mathfrak{t}}_{0}+\mathfrak{v}_{\text {en }}\right)\left[\left(\sum_{n=1}^{K} b_{n, k_{2}, \ldots, k_{N}}^{(j)} \phi_{n}^{(j)}\right),\left(\sum_{n=1}^{K} b_{n, k_{2}, \ldots, k_{N}}^{(j)} \phi_{n}^{(j)}\right)\right] \\
\geq\left(\tilde{\mathfrak{t}}_{0}+\mathfrak{v}_{\mathrm{en}}\right)\left[\left(\sum_{n=1}^{K} b_{n, k_{2}, \ldots, k_{N}} \phi_{n}\right),\left(\sum_{n=1}^{K} b_{n, k_{2}, \ldots, k_{N}} \phi_{n}\right)\right] .
\end{gathered}
$$

Furthermore, as in [29], Fatou's lemma yields

$$
\begin{aligned}
& \liminf _{j \rightarrow \infty} \int_{\mathbb{R}^{3}} \int_{\mathbb{R}^{3}} \frac{\left(\sum_{1 \leq m, n \leq K} b_{m, n, k_{3}, \ldots, k_{N}}^{(j)} \phi_{m}^{(j)}(x) \phi_{n}^{(j)}(y)\right)^{2}}{|x-y|} d x d y . \\
& \geq \int_{\mathbb{R}^{3}} \int_{\mathbb{R}^{3}} \frac{\left(\sum_{1 \leq m, n \leq K} b_{m, n, k_{3}, \ldots, k_{N}} \phi_{m}(x) \phi_{n}(y)\right)^{2}}{|x-y|} d x d y .
\end{aligned}
$$

and we easily conclude.

\section{The MC Hartree-Fock type EQUATions}

The Euler-Lagrange equations of the minimization problem in Definition 4.2 can be derived in the same way as the HF equations, and one then obtains the multi-configurative $\mathrm{HF}$ type equations [38, 28, 17, 29], which take the following form, for $1 \leq k \leq K$,

$$
\begin{aligned}
d_{k}\left(\widetilde{T}_{0}+V_{\mathrm{en}}\right) \phi_{k}+\sum_{k l m n} c_{k l m n}\left(\phi_{l} \phi_{m} * \frac{1}{|x|}\right) \phi_{n} & =\sum_{l=1}^{K} \lambda_{k l} \phi_{l}, \\
T_{\phi} \cdot a & =\zeta \cdot a
\end{aligned}
$$

where the $c_{l k m n}$ are real numbers which can be expressed via $a$ and the $\lambda_{k l}$ are Lagrange multipliers corresponding to the orthonormality of the $\phi_{n}$. The equations consist of $K$ HF type nonlinear partial differential equations for $\phi_{n}$, coupled to a Schrödinger type linear system for the $a$ arising as the Euler-Lagrange equations for the $a_{J}$ with $\zeta=\mathcal{E}_{K, N}(a, \boldsymbol{\phi})$ being the multiplier, and $T_{\boldsymbol{\phi}}=\left\{\left\langle\boldsymbol{\phi}_{I}, T_{N, \boldsymbol{Z}, \alpha} \boldsymbol{\phi}_{J}\right\rangle\right\}_{I, J}$ being the $\left(\begin{array}{l}K \\ N\end{array}\right) \times\left(\begin{array}{l}K \\ N\end{array}\right)$ matrix of the Schrödinger operator $T_{N, \boldsymbol{Z}, \alpha}$ on $\operatorname{span}\left\{\phi_{J}: J\right\}$. The $d_{k}=\sum_{J \mid k \in J}\left|a_{J}\right|^{2}$ are the so-called occupation numbers. For our purpose it is convenient to write the HF type equations (6.1) as in (11.1)-(11.2) (see Theorem 11.1), wherein $\boldsymbol{D}$ and $\boldsymbol{E}_{\boldsymbol{\phi}}$ are defined in (4.9)-(4.10), and $\Lambda$ is the Lagrange multiplier matrix corresponding to the constraints $\left\langle\phi_{m}, \phi_{n}\right\rangle_{L^{2}\left(\mathbb{R}^{3}\right)}=\delta_{m n}$. This form was given by Lewin [29] (but similar calculations are found in many books, 
e.g., [38]).

Define

$$
\mathrm{J}_{K, N}=\{J \subset\{1, \ldots, K\}:|J|=N\} .
$$

By choosing a set $\mathcal{J} \subset \mathrm{J}_{K, N}$ and restricting the energy $\mathcal{E}_{K, N}$ to the submanifold

$$
\mathcal{C}_{\mathcal{J}, K, N}=\left\{(a, \Phi) \in \mathcal{C}_{K, N}: \forall J \notin \mathcal{J}, \quad a_{J}=0\right\} \subset \mathcal{C}_{K, N},
$$

we obtain a partial MC method of rank $K$ with corresponding ground state energy defined by

$$
E_{\mathcal{J}, K, N}=\inf _{\mathcal{C}_{\mathcal{J}, K, N}} \mathcal{E}_{K, N}
$$

Then the critical points of $\mathcal{E}_{K, N}$ on $\mathcal{C}_{\mathcal{J}, K, N}$ will be solutions of the equations

$$
\left.\begin{array}{rl}
\left(\left(\widetilde{T}_{0}+V_{\text {en }}\right) \boldsymbol{D}+2 \boldsymbol{E}_{\Phi}\right) \Phi+\Lambda \cdot \Phi & =0, \\
T_{\Phi}^{\mathcal{J}} \cdot a_{\mathcal{J}} & =\zeta \cdot a_{\mathcal{J}} .
\end{array}\right\}
$$

with $a_{\mathcal{J}}=\left\{a_{J}\right\}_{J \in \mathcal{J}}$ and $T_{\Phi}^{\mathcal{J}}=\left\{\left\langle\Phi_{I}, T_{N, \boldsymbol{Z}, \alpha} \Phi_{J}\right\rangle\right\}_{I, J \in \mathcal{J}}$ being the $|\mathcal{J}| \times$ $|\mathcal{J}|$ matrix of the Schrödinger operator $T_{N, \boldsymbol{Z}, \alpha}$ on $\operatorname{span}\left\{\Phi_{J}: J \in \mathcal{J}\right\}$.

The "natural MC methods" (as pioneered by Löwdin) and used by chemists is defined as follows [32, 38, 28, 23, 29]:

Definition 6.1. A natural multi-configurative method of rank $K$ is defined by a $\mathcal{J} \subset \mathrm{J}_{K, N}$ for which:

(i) There exists a $(a, \Phi) \in \mathcal{C}_{\mathcal{J}, K, N}$ of rank $K$;

(ii) for every $(a, \Phi) \in \mathcal{C}_{\mathcal{J}, K, N}$ there exists

$$
\boldsymbol{U} \in \mathcal{O}_{\mathcal{J}}=\left\{\boldsymbol{U} \in \mathcal{O}_{K}(\mathbb{R}): \boldsymbol{U} \cdot \mathcal{C}_{\mathcal{J}, K, N} \subset \mathcal{C}_{\mathcal{J}, K, N}\right\}
$$

such that $\boldsymbol{D}^{\prime}$, corresponding to $\left(a^{\prime}, \Phi^{\prime}\right)=\boldsymbol{U}(a, \Phi)$, is a diagonal matrix.

Definition 6.1(ii) asserts that any wave function can be written in terms of its natural orbitals.

Assumption 6.2. Let $\mathcal{J}$ define a natural MC method of rank $K$ and let $\left\{\left(a^{(j)}, \phi^{(j)}\right)\right\}_{j=1}^{\infty}$ be a sequence in $\mathcal{C}_{\mathcal{J}, K, N}$ such that

(i) $\lim _{j \rightarrow \infty} \mathcal{E}_{K, N}\left(a^{(j)}, \phi^{(j)}\right)=l$,

(ii) For each $J \in \mathcal{J}$,

$$
\lim _{j \rightarrow \infty} \mathrm{d}_{\phi} \mathcal{E}_{K, N}\left(a^{(j)}, \boldsymbol{\phi}^{(j)}\right)=\mathbf{0} \quad \text { and } \quad \lim _{j \rightarrow \infty} \partial_{a} \mathcal{E}_{K, N}\left(a^{(j)}, \boldsymbol{\phi}^{(j)}\right)=\mathbf{0} .
$$

(iii) There exists $m$ and a sequence of positive real numbers $\left\{\delta^{(j)}\right\}_{j}$ with $\delta^{(j)} \searrow 0$ such that for every $j \mathrm{~d}_{\phi}^{2} \mathcal{E}_{K, N}\left(a^{(j)}, \phi^{(j)}\right)$ has at most $m$ eigenvalues below $-\delta^{(j)}$. 
Mixed configuration. Assume $\boldsymbol{\Psi}=\sum a_{J} \boldsymbol{\Phi}_{J}$ where $\left\{\phi_{1}, \ldots, \phi_{K}\right\}$ is a set of orthonormal orbitals, $\boldsymbol{\Phi}_{J}=\phi_{j_{1}} \wedge \cdots \wedge \phi_{j_{N}}$, and the multiindex $J=\left(j_{1}, \ldots, j_{N}\right)$ runs over $1 \leq j_{1}<\cdots<j_{N} \leq K$. The following lemma is attributed to Friesecke [18].

Lemma 6.3. Let $\boldsymbol{\Psi}, \boldsymbol{\Phi}_{J}$ and $J$ be as above. Suppose $I=\left(i_{1}, \ldots, i_{N}\right)$ is any multi-index such that $a_{I} \neq 0$. Then, either

$$
\left\langle\boldsymbol{\Psi}, T_{N, \boldsymbol{Z}, \alpha} a^{\dagger}(\chi) a^{\dagger}(\eta) a\left(\phi_{i_{2}}\right) a\left(\phi_{i_{1}}\right) \boldsymbol{\Phi}_{I}\right\rangle=0
$$

for any $\chi, \eta$ satisfying $\left\langle\chi, \phi_{n}\right\rangle=\left\langle\eta, \phi_{n}\right\rangle=0$ for all $n=1, \ldots, K$, or $\boldsymbol{\Psi}$ is not a ground state of $T_{N, \boldsymbol{Z}, \alpha}$.

Since we shall refer to the contents of its proof later, we give the short proof of this lemma.

Proof. We let $\boldsymbol{\Psi}^{\prime}:=a^{\dagger}(\eta) a\left(\phi_{i_{2}}\right) a\left(\phi_{i_{1}}\right) \boldsymbol{\Phi}_{I}$ and also $C^{2}=\left\langle\boldsymbol{\Psi}^{\prime}, \boldsymbol{\Psi}^{\prime}\right\rangle$. Then $C \neq 0$ for $\chi \neq \eta$, and we may define $\widetilde{\Psi}=\Psi^{\prime} / C$. Moreover, for any $\epsilon \in(0,1)$, we define $\boldsymbol{\Psi}_{\epsilon}=(\epsilon / C) \widetilde{\Psi}+(1-\epsilon)^{1 / 2} \boldsymbol{\Psi}$. Then the expectation of the energy for $T_{N, \boldsymbol{Z}, \alpha}$ in the state $\boldsymbol{\Psi}_{\epsilon}$ can be expanded in $\epsilon$ :

$$
\begin{aligned}
\mathcal{E}\left(\boldsymbol{\Psi}_{\epsilon}\right)=\mathcal{E}(\boldsymbol{\Psi})+ & \epsilon\left(1-\epsilon^{2}\right)^{1 / 2}\left(\left\langle\boldsymbol{\Psi}, T_{N, \boldsymbol{Z}, \alpha} \widetilde{\boldsymbol{\Psi}}\right\rangle+\text { c.c. }\right) \\
+ & \epsilon^{2}(\mathcal{E}(\widetilde{\boldsymbol{\Psi}})-\mathcal{E}(\boldsymbol{\Psi}))
\end{aligned}
$$

By taking $\epsilon$ small enough, we can ensure that the $\epsilon^{2}$ term is dominated y the $\epsilon\left(1-\epsilon^{2}\right)^{1 / 2}$ term and, consequently, the right-hand side is strictly less than $\mathcal{E}(\boldsymbol{\Psi})$ provided there exist $\chi$ and $\eta$ such that $\mathcal{E}(\widetilde{\boldsymbol{\Psi}})-\mathcal{E}(\boldsymbol{\Psi})$ is negative. The latter inequality holds if and only if there exist $\chi$ and $\eta$ such that $\left\langle\boldsymbol{\Psi}, T_{N, \boldsymbol{Z}, \alpha} \widetilde{\boldsymbol{\Psi}}\right\rangle \neq 0$ because, in this case, we can just multiply $\chi$ by a trivial phase factor.

\section{The QUASI-RElativistiC FoCK OPERATOR}

Herein we introduce the quasi-relativistic Fock operator.

Lemma 7.1. Assume $Z_{\text {tot }}<Z_{\mathrm{c}}$. Let

$$
K^{\mathrm{xc}}\left(x, x^{\prime}\right)=\frac{\mathcal{D}\left(x, x^{\prime}\right)}{\left|x-x^{\prime}\right|}
$$

be the integral kernel of the exchange operator $K^{\mathrm{xc}}$. Then the unique self-adjoint operator $F$ associated with the differential expression

$$
\alpha^{-1} \tilde{T}_{0} \phi+\alpha^{-1} V_{\mathrm{en}} \phi+\rho * \frac{1}{|x|} \phi-K^{\mathrm{xc}} \phi,
$$


is generated by the sesquilinear form

$$
\begin{aligned}
& \mathfrak{f}[\phi, \psi]=\alpha^{-1} \tilde{\mathfrak{t}}_{0}[\phi, \psi]+\alpha^{-1} \int_{\mathbb{R}^{3}} V_{\text {en }}(x) \phi(x) \bar{\psi}(x) \mathrm{d} x \\
& +\int_{\mathbb{R}^{3}} \int_{\mathbb{R}^{3}} \frac{\rho(x) \phi(y) \bar{\psi}(y)}{|x-y|} \mathrm{d} x \mathrm{~d} y-\int_{\mathbb{R}^{3}} \int_{\mathbb{R}^{3}} \mathcal{D}(x, y) \frac{\phi(y) \bar{\psi}(x)}{|x-y|} \mathrm{d} y \mathrm{~d} x .
\end{aligned}
$$

Proof. Bear in mind the definitions of $\mathfrak{t}_{0}, \tilde{\mathfrak{t}}_{0}$ and $\mathfrak{v}_{\text {en }}$ from Section 3. Define $\mathfrak{v}_{\rho *(1 /|x|)}$ as the third form on the right-hand side of (7.3). Then (3.1) yields the estimate

$$
\mathfrak{v}_{\rho *(1 /|x|)}[\phi, \phi] \leq C\left(Z_{\mathrm{c}}, N\right) \mathfrak{t}_{0}[\phi, \phi] .
$$

Under the hypothesis, we already know from Section 3 that the quadratic form $\mathfrak{t}_{0}+\mathfrak{v}_{\text {en }}$ is nonnegative on $\mathbf{H}^{1 / 2}\left(\mathbb{R}^{3}\right)$. Evidently, $\mathfrak{v}_{\rho *(1 /|x|)}$ is a nonnegative form and, consequently, $\tilde{\mathfrak{f}}=\mathfrak{t}_{0}+\mathfrak{v}_{\mathrm{en}}+\mathfrak{v}_{\rho *(1 /|x|)}$ is a nonnegative form on $\mathbf{H}^{1 / 2}\left(\mathbb{R}^{3}\right)$. Closedness of the nonnegative quadratic form $\tilde{\mathfrak{f}}$ is equivalent to lower semicontinuity of $\tilde{\mathfrak{f}}$ on $\mathbf{H}^{1 / 2}\left(\mathbb{R}^{3}\right)$. In fact, $\tilde{\mathfrak{f}}$ is continuous. Indeed, (3.1), respectively (7.4), enables us to show continuity of the second, respectively the third term, in $\tilde{\mathfrak{f}}$. For instance, we consider $\mathfrak{v}_{\text {en }}$ and assume that $\phi_{j} \rightarrow \phi$ in $\mathbf{H}^{1 / 2}\left(\mathbb{R}^{3}\right)$. Then an application of Hölder's inequality and (3.1) yields

$$
\begin{aligned}
& \left|\mathfrak{v}_{\mathrm{en}}\left[\phi_{j}\right]-\mathfrak{v}_{\mathrm{en}}[\phi]\right| \leq \int_{\mathbb{R}^{3}}\left|V_{\mathrm{en}}\right|\left|\phi_{j}-\phi \| \phi_{j}+\phi\right| \mathrm{d} x \\
& \leq c\left(\int_{\mathbb{R}^{3}}\left|V_{\mathrm{en}}\right|\left|\phi_{j}-\phi\right|^{2} \mathrm{~d} x\right)^{1 / 2} \leq C\left\|\phi_{j}-\phi\right\|_{\mathbf{H}^{1 / 2}\left(\mathbb{R}^{3}\right)} .
\end{aligned}
$$

We conclude that $\tilde{\mathfrak{f}}$ is a closed quadratic form on $\mathbf{H}^{1 / 2}\left(\mathbb{R}^{3}\right)$. The first representation theorem [12, Theorem VI.2.4] informs us that the nonnegative, closed form $\tilde{\mathfrak{f}}$ is associated to a unique self-adjoint operator, say $\widetilde{F}$. Furthermore, the exchange operator $K^{\mathrm{xc}}$ is a Hilbert-Schmidt operator. Indeed, using, in this particular order, the weak Young inequality, the Hölder inequality and (3.1) we find that $K^{\mathrm{xc}}\left(x, x^{\prime}\right) \in$ $L^{2}\left(\mathbb{R}^{3} \times \mathbb{R}^{3}\right)$. It is clear that the form $\tilde{\mathfrak{f}}[\cdot, \cdot]-\alpha^{-1}\langle\cdot, \cdot\rangle_{L^{2}}-\left\langle K^{\mathrm{xc}} \cdot, \cdot\right\rangle_{L^{2}}$ is closed and, once again applying the first representation theorem, we obtain a unique self-adjoint operator $F$ associated with the form in (7.3).

\section{LOWER SPECTRAL BOUND}

We will later need the following spectral result. 
Lemma 8.1. Assume $\vartheta<Z_{\text {tot }}<Z_{\mathrm{c}}$, and let $\rho \in L^{1}\left(\mathbb{R}^{3}\right) \cap L^{4 / 3}\left(\mathbb{R}^{3}\right)$ such that $\int_{\mathbb{R}^{3}} \rho \mathrm{d} x<\vartheta$. Define the quasi-relativistic Schrödinger operator

$$
T=\alpha^{-1} \widetilde{T}_{0}+\alpha^{-1} V_{\text {en }}+\rho * \frac{1}{|x|} .
$$

Then, for any $\kappa \geq 1$ and any $0 \leq \vartheta<Z_{\text {tot }}$, there exists $\epsilon_{\kappa, \vartheta}>0$ such that

$$
\operatorname{Coun}\left(-\epsilon_{n, \vartheta} ; T\right) \geq \kappa
$$

Proof. By a minor modification of [21, p 291], which carries over the result (3.5) from the one-nucleus to the many-nuclei case, we deduce that the essential spectrum of $\tilde{T}_{0}+V_{\text {en }}$ equals the semiaxis $[0, \infty)$. Next, a standard perturbation argument and an application of Weyl's essential spectrum theorem proves that $\sigma_{\text {ess }}(T)=[0, \infty)$. Let $\mathfrak{t}_{\mu}$ denote the quadratic form defined by

$$
\alpha^{-1} \tilde{\mathfrak{t}}_{0}[\phi, \phi]+\int_{\mathbb{R}^{3}}\left(\alpha^{-1} V_{\text {en }}+\rho * \frac{1}{|x|}\right)|\phi(x)|^{2} \mathrm{~d} x .
$$

For any $\kappa \geq 1$ and any $0 \leq \vartheta \leq Z_{\text {tot }}$ we construct a $\kappa$-dimensional subspace $\mathcal{H}_{\kappa, \vartheta}$ in $\mathbf{H}^{1 / 2}\left(\mathbb{R}^{3}\right)$ such that

$$
\mathfrak{t}[\phi, \phi]<-\epsilon_{\kappa, \vartheta}<0
$$

for all $L^{2}$-normalized $\phi \in \mathcal{H}_{\kappa, \vartheta}$. We note that

$$
\alpha^{-1} \tilde{\mathfrak{t}}_{0}[\phi, \phi] \leq C \int_{\mathbb{R}^{3}}|\nabla \phi|^{2} \mathrm{~d} x .
$$

As a consequence, by selecting a $\kappa$-dimensional subspace of normalized, radially symmetric functions in $C_{0}^{\infty}\left(\mathbb{R}^{3}\right)$, we can construct a subspace $\mathcal{H}_{\kappa, \vartheta}$ of functions satisfying (8.2) by repeating the arguments in [31, Lemma II.1] (see also [13, Lemma 6.1]). Then the assertion follows by an application of Glazman's Lemma (see, e.g., [36, Lemma A.3]).

Within the nonrelativistic context a similar result was first given by Lions [31, Lemma II.1].

\section{Convergence of Palais-Smale type sequences}

In this section we give the main auxiliary result that will be used in the proof of Theorem 10.1 and Theorem 11.1.

Proposition 9.1. Assume that $l \in \mathbb{R}, N-1<Z_{\text {tot }}<Z_{\text {c }}$, and $K \geq$ $N$. If a sequence $\left(a^{(j)}, \phi^{(j)}\right)$ satisfies Assumption 6.2, then there exists $\widetilde{K} \in[N, K]$ such that, up to extracting subsequences and up to unitary transformations $\boldsymbol{U}^{(j)} \in \mathcal{O}_{K}(\mathbb{R})$, the following holds: 
1. $\mathbf{D}^{(j)}=\operatorname{diag}\left(d_{1}^{(j)}, \ldots, d_{K}^{(j)}\right)$ for all $j \in \mathbb{N}$.

2. For each $n=1, \ldots, \widetilde{K}, d_{n}^{(j)} \rightarrow d_{n}>0$ and $\phi_{n}^{(j)} \rightarrow \phi_{n}$ strongly in $\mathbf{H}^{1 / 2}\left(\mathbb{R}^{3}\right)$.

3. For each $n=\widetilde{K}+1, \ldots, K, d_{n}^{(j)} \rightarrow 0$ and $\sqrt{d_{n}^{(j)}} \phi_{n}^{(j)} \rightarrow 0$ strongly in $\mathbf{H}^{1 / 2}\left(\mathbb{R}^{3}\right)$.

4. If $\widetilde{\mathcal{J}}=\mathcal{J} \cap \mathrm{J}_{\widetilde{K}, N}, \tilde{a}=\left(a_{J}\right)_{J \in \mathrm{J}_{\widetilde{K}, N}}$ and $\widetilde{\boldsymbol{\phi}}=\left(\phi_{1}, \ldots, \phi_{\widetilde{K}}\right)$, then $(\tilde{a}, \widetilde{\boldsymbol{\phi}})$ is a critical point of $\mathcal{E}_{\widetilde{K}, N}$ on $\mathcal{C}_{\widetilde{\mathcal{J}}, \widetilde{K}, N}$ and it is a solution to the equations in (6.3), wherein $\Lambda>0$.

5. If $\mathcal{J}=\mathrm{J}_{K, N}$, then either $\widetilde{K}=K-1$ or $\widetilde{K}=K$.

6. If $\boldsymbol{\Psi}^{\prime}$ is the wave function corresponding to $\left(a^{\prime}, \boldsymbol{\Phi}^{\prime}\right)$, then $\boldsymbol{\Psi}^{(j)} \rightarrow \boldsymbol{\Psi}$ strongly in $\mathbf{H}_{\mathrm{a}}^{1 / 2}\left(\mathbb{R}^{3 N}\right)$.

Proof.

It follows from the hypotheses that, without loss of generality, we may suppose that $\boldsymbol{D}^{j}=\operatorname{diag}\left(d_{1}^{(j)}, \ldots, d_{K}^{(j)}\right), d_{n}^{(j)} \in[0,1]$, and $\left\{d_{n}^{(j)}\right\}_{n=1}^{K}$ are monotonically decreasing as $n$ grows. In particular, as $j \rightarrow \infty$ one has $d_{n}^{(j)} \rightarrow d_{n}$ for some $d_{n}$ and hence there exists some $a_{J}$ such that $a_{J}^{(j)} \rightarrow a_{J}$ for every $J \in \mathcal{J}$. If $K=N$, then $d_{n}^{(j)}=1$ for every $n=1, \ldots, N$ and $j \in \mathbb{N}$. An application of (3.1) and the first order information in Assumption 6.2 give us that

$$
\sum_{n=1}^{K} d_{n}^{(j)} \mathfrak{t}_{0}\left[\phi_{n}^{(j)}, \phi_{n}^{(j)}\right] \leq \mathrm{const}\left[1-\frac{Z_{\mathrm{tot}}}{Z_{\mathrm{c}}}\right]^{-1} .
$$

We deduce that either $d_{n}=0$ or $\left\{\phi_{n}^{(j)}\right\}$ is bounded in $\mathbf{H}^{1 / 2}\left(\mathbb{R}^{3}\right)$. We define $\widetilde{K} \geq N$ to be the smallest integer satisfying that $d_{\widetilde{K}+1}=0$ and $d_{\widetilde{K}} \neq 0$. Next we prove that, for any $m=\widetilde{K}+1, \ldots, K$, (delete maybe) $d_{m}^{(j)} \rightarrow 0$ and $\sqrt{d_{m}^{(j)}} \phi_{m}^{(j)} \rightarrow 0$ strongly in $\mathbf{H}^{1 / 2}\left(\mathbb{R}^{3}\right)$. Since $\left\{d_{m}^{(j)}\left\|\phi_{m}^{(j)}\right\|\right\}_{j}$ is bounded, we infer that, after possibly passing to a subsequence, $\sqrt{d_{m}^{(j)}} \phi_{m}^{(j)} \rightarrow 0$ weakly in $\mathbf{H}^{1 / 2}\left(\mathbb{R}^{3}\right)$. We may suppose that $d_{m}^{(j)} \rightarrow 0$ (as $j \rightarrow \infty)$. The sequence $\left\{\phi_{m}^{(j)}\right\}_{j}$ is bounded in $L^{2}\left(\mathbb{R}^{3}\right)$ and, therefore, we may assume (after possibly extracting a subsequence) that $\sqrt{d_{m}^{(j)}} \phi_{m}^{(j)} \rightarrow$ 0 in $L^{2}\left(\mathbb{R}^{3}\right)$. Although the functional $\mathcal{E}$ is not quadratic with respect to the orbitals it is, however, quadratic in $a_{J}$. Explicitly, for some sequences $\mathcal{E}_{1}^{(j)}, \mathcal{E}_{2}^{(j)}, \mathcal{E}_{3}^{(j)}$, we have that

$$
\mathcal{E}_{K, N}\left(a^{(j)}, \phi^{(j)}\right)=\left(\sum_{n \in J} \tilde{\mathfrak{t}}_{0}\left[\phi_{n}^{(j)}, \phi_{n}^{(j)}\right]\right)\left(a_{J \mid m}^{(j)}\right)^{2}+\mathcal{E}_{1}^{(j)}\left(a_{J \mid m}^{(j)}\right)^{2}+\mathcal{E}_{2}^{(j)} a_{J \mid m}^{(j)}+\mathcal{E}_{3}^{(j)},
$$


where $J \mid m$ indicates that $J$ contains $m$. Using the above-mentioned convergence properties it is easy to see that the terms $\mathcal{E}_{1}^{(j)}\left(a_{J}^{(j)}\right)^{2}$ and $\mathcal{E}_{2}^{(j)} a_{J}^{(j)}$ tend to zero as $j \rightarrow \infty$. Using the first order information in Assumption 6.2 yields

$$
\begin{aligned}
\mathrm{d}_{a_{J \mid m}} \mathcal{E}_{K, N}\left(a^{(j)}, \phi^{(j)}\right)= & 2 \sum_{n \in J} \tilde{\mathfrak{t}}_{0}\left[\phi_{n}^{(j)}, \phi_{n}^{(j)}\right] a_{J \mid m}^{(j)} \\
& +2 \mathcal{E}_{1}^{(j)} a_{J \mid m}^{(j)}+\mathcal{E}_{2}^{(j)}+\lambda_{J \mid m}^{(j)} a_{J \mid m}^{(j)} \longrightarrow 0,
\end{aligned}
$$

where $\lambda_{J \mid m}^{(j)}$ is a sequence of Lagrange multipliers. We multiply by $a_{J \mid m}^{(j)}$ and, as $j \rightarrow \infty$, get that

$$
\mathfrak{t}_{0}\left[\phi_{n}^{(j)}, \phi_{n}^{(j)}\right]\left(a_{J \mid m}^{(j)}\right)^{2} \longrightarrow 0
$$

Now, by definition

$$
d_{m}^{(j)}=\sum_{J \mid m}\left(a_{J}^{(j)}\right)^{2}
$$

and we deduce that

$$
\sqrt{d_{m}^{(j)}} \phi_{m}^{(j)} \longrightarrow 0 \quad \text { strongly }
$$

in $\mathbf{H}^{1 / 2}\left(\mathbb{R}^{3}\right)$.

For all $J \in \mathrm{J}_{\widetilde{K}, N}$ and $\widetilde{\phi}^{(j)}=\left(\phi_{1}^{(j)}, \ldots, \phi_{\widetilde{K}}^{(j)}\right)^{\mathrm{T}}$, we set $\tilde{\mathcal{J}}=\mathcal{J} \cap \mathrm{J}_{\widetilde{K}, N}$ and define

$$
\tilde{a}_{J}^{(j)}=\frac{a_{J}^{(j)}}{\sqrt{\sum_{I \subset\{1, \ldots, \widetilde{K}\}}\left(a_{I}^{(j)}\right)^{2}}} .
$$

Then the new sequence $\left\{\left(\tilde{a}^{(j)}, \widetilde{\phi}^{(j)}\right)\right\}$ fulfills the hypotheses (i)-(iii) on $\mathcal{C}_{\widetilde{\mathcal{J}}, \widetilde{K}, N}$. We will denote this sequence by $\left\{\left(a^{(j)}, \phi^{(j)}\right)\right\}$ in the sequel. In a standard way the first-order information in Assumption 6.2 implies that

$$
\left(\left(\widetilde{T}_{0}+V_{\mathrm{en}}\right) \boldsymbol{D}^{(j)}+2 \boldsymbol{E}_{\boldsymbol{\phi}^{(j)}}\right) \boldsymbol{\phi}^{(j)}+\Lambda^{(j)} \boldsymbol{\phi}^{(j)} \rightarrow 0
$$

in $L^{2}\left(\mathbb{R}^{3}\right)^{\widetilde{K}}$. We proceed to extract a few subsequences. We begin by diagonalizing $\Lambda^{(j)}$, with $\operatorname{diag}\left(\lambda_{1}^{(j)}, \ldots, \lambda_{\widetilde{K}}^{(j)}\right)$, via an appropriate choice of orthogonal matrices $\boldsymbol{U}^{(j)}$. Henceforth we study the sequence $\left\{\boldsymbol{U}^{(j)}\left(a^{(j)}, \boldsymbol{\phi}^{(j)}\right)\right\}$; we denote it by $\left\{\left(\tilde{a}^{(j)}, \widetilde{\boldsymbol{\phi}}^{(j)}\right)\right\}$ in the sequel. For this 
purpose we introduce

$$
\begin{aligned}
\rho_{n}^{(j)}= & \frac{\tilde{N}}{\tilde{d}_{n n}^{(j)}} \sum_{k_{3}, \ldots, k_{N}}\left(\sum_{k} \tilde{b}_{n, k, k_{3}, \ldots, k_{N}} \tilde{\phi}_{k}^{(j)}\right)^{2} \\
\mathfrak{a}_{n}^{(j)}[\psi, \psi]= & \sum_{k_{3}, \ldots, k_{N}} \int_{\mathbb{R}^{3}} \int_{\mathbb{R}^{3}} \frac{1}{\left|x-x^{\prime}\right|}\left(\psi(x) \sum_{k} \tilde{b}_{n, k, k_{3}, \ldots, k_{N}} \tilde{\phi}_{k}^{(j)}(x)\right) \\
& \times\left(\psi\left(x^{\prime}\right) \sum_{k} \tilde{b}_{n, k, k_{3}, \ldots, k_{N}} \tilde{\phi}_{k}^{(j)}\left(x^{\prime}\right)\right) \mathrm{d} x \mathrm{~d} x^{\prime} .
\end{aligned}
$$

By using the second-order information on the sequence, see Assumption 6.2(iii), and the form $\mathfrak{f}$. in Lemma 7.1, we get that

$$
\tilde{d}_{n n}^{(j)} \mathfrak{f}_{\rho_{n}^{(j)}}[\psi, \psi]+\left(\lambda_{n}^{(j)}+\tilde{d}_{n n}^{(j)} \delta_{n}^{(j)}\right)\|\psi\|_{L^{2}\left(\mathbb{R}^{3}\right)}^{2}-\tilde{N} \mathfrak{a}_{n}^{(j)} \geq 0
$$

for every $n=1, \ldots, \widetilde{K}$ and every $\psi$ belonging to a closed subspace $\mathcal{H}^{(j)}$ of $\mathbf{H}^{1 / 2}\left(\mathbb{R}^{3}\right)$ with finite codimension at most $\widetilde{K}+m$. The numbers $\delta_{n}^{(j)}$ in (9.3) tend to zero in the standard Euclidean metric for each fixed $n$. Since $\mathfrak{a}_{n}^{(j)}$ is nonnegative, we infer that

$$
\mathfrak{f}_{\rho_{n}^{(j)}}[\psi, \psi]+\left(\frac{\lambda_{n}^{(j)}}{\tilde{d}_{n n}^{(j)}}+\delta_{n}^{(j)}\right)\|\psi\|_{L^{2}\left(\mathbb{R}^{3}\right)}^{2} \geq 0
$$

for all $\psi \in \mathcal{H}^{(j)}$ (after perhaps going to a subsequence). By invoking Lemma 2.1 we deduce that the quasi-relativistic Schrödinger operator

$$
T_{n}^{(j)}=\widetilde{T}_{0}+V_{\mathrm{en}}+\rho_{n}^{(j)} * \frac{1}{\left|x-x^{\prime}\right|}
$$

has at most $\widetilde{K}+m$ eigenvalues strictly less than $-\left(\lambda_{n}^{(j)} / \tilde{d}_{n n}^{(j)}\right)-\delta_{n}^{(j)}$. Moreover, since

$$
\int_{\mathbb{R}^{3}} \rho_{n}^{(j)} d x=\frac{\tilde{N}}{\tilde{d}_{n n}^{(j)}} \sum_{i, k, k_{3}, k_{4}, \ldots, k_{N}}\left(\tilde{b}_{i, k, k_{3}, \ldots, k_{N}}\right)^{2}=N-1<Z_{\mathrm{tot}}
$$

Lemma 8.1 ensures that there exists a $\delta>0$ (independent of $j$ ) such that $T_{n}^{(j)}$ has at least $\widetilde{K}+m$ eigenvalues strictly below $-\delta$. As a consequence, we infer that

$$
\frac{\lambda_{n}^{(j)}}{\tilde{d}_{n n}^{(j)}}+\delta_{n}^{(j)} \geq \delta, \quad \forall j
$$


Since $\delta_{n}^{(j)} \rightarrow 0$ as $j \rightarrow \infty$ and $\widetilde{\mathbf{D}}^{(j)}=\boldsymbol{U}_{(j)} \mathbf{D}^{(j)} \boldsymbol{U}_{(j)}^{\mathrm{T}} \geq d \boldsymbol{I}_{\widetilde{K}}$, we deduce that, for $j$ large enough,

$$
\lambda_{n}^{(j)} \geq \frac{\tilde{d}_{n n}^{(j)} \delta}{2} \geq \frac{d \delta}{2}=: \lambda>0, \quad \forall n .
$$

Consequently, $\Lambda^{(j)} \geq \lambda \boldsymbol{I}_{\widetilde{K}}$. In view of (9.2) we infer that $\left\{\Lambda^{(j)}\right\}$ is bounded. In particular, up to extracting a subsequence, we deduce that $\Lambda^{(j)} \rightarrow \Lambda>0$. From above, $d_{n}^{(j)} \geq d>0$ and this implies that $\left\{\phi_{n}^{(j)}\right\}_{j \in \mathbb{N}}$ are bounded in $\left(\mathbf{H}^{1 / 2}\left(\mathbb{R}^{3}\right)\right)^{K}$ and, as a consequence, we may suppose that $\phi_{n}^{(j)}$ converges weakly in $\mathbf{H}^{1 / 2}\left(\mathbb{R}^{3}\right.$ ) (and a.e. in $\mathbb{R}^{3}$ ) to some $\phi_{n}$. We proceed to showing that $\phi_{n}^{(j)} \rightarrow \phi_{n}$ strongly in $\mathbf{H}^{1 / 2}\left(\mathbb{R}^{3}\right)$. By going to the limit in (9.2), we get that

$$
\left(\left(\widetilde{T}_{0}+V_{\mathrm{en}}\right) \boldsymbol{D}+2 \boldsymbol{E}_{\boldsymbol{\phi}}\right) \cdot \boldsymbol{\phi}+\Lambda \cdot \boldsymbol{\phi}=0
$$

which, by invoking the same argument as in the proof of Lemma 5.1, yields

$$
\begin{aligned}
& \liminf _{j \rightarrow \infty}\left\langle\Lambda^{(j)} \boldsymbol{\phi}^{(j)}, \boldsymbol{\phi}^{(j)}\right\rangle \\
& \quad=-\liminf _{j \rightarrow \infty}\left\langle\left(\left(\widetilde{T}_{0}+V_{\mathrm{en}}\right) \boldsymbol{D}^{(j)}+2 \boldsymbol{E}_{\boldsymbol{\phi}^{(j)}}\right) \boldsymbol{\phi}^{(j)}, \boldsymbol{\phi}^{(j)}\right\rangle \\
& \leq-\left\langle\left(\left(\widetilde{T}_{0}+V_{\mathrm{en}}\right) \boldsymbol{D}+2 \boldsymbol{E}_{\boldsymbol{\phi}}\right) \boldsymbol{\phi}, \boldsymbol{\phi}\right\rangle=\langle\Lambda \boldsymbol{\phi}, \boldsymbol{\phi}\rangle .
\end{aligned}
$$

Hence $\boldsymbol{\phi}^{(j)} \rightarrow \boldsymbol{\phi}$ in $\left(L^{2}\left(\mathbb{R}^{3}\right)\right)^{\tilde{K}}$, and in $\left(\mathbf{H}^{1 / 2}\left(\mathbb{R}^{3}\right)\right)^{\tilde{K}}$ as well. The convergence of $\left\{\boldsymbol{\Psi}^{(j)}\right.$ follows immediately. Finally we show that if $\mathcal{J}=\mathrm{J}_{K, N}$ then either $\widetilde{K}=K$ or $\widetilde{K}=K-1$. The result follows if we establish the inequality $E_{N}^{K+2}<E_{N}^{K}$. To prove this inequality we can adopt the reasoning in [18]. Therein it is demonstrated that it is not possible for $\boldsymbol{\Psi}$ to satisfy the equations (11.1)-(11.2) of rank $K$ if none of the trial functions $\boldsymbol{\Psi}_{\epsilon}$ in the proof of Lemma 6.3, whose rank does not exceed $K+2$, has lower energy than $\boldsymbol{\Psi}$. Bearing in mind that a (" $(K-N)$-fold excited") ground state is a minimizer of $\mathcal{E}(\boldsymbol{\Psi})$ among all normalized antisymmetric wavefunctions which satisfy $\operatorname{rank}(\boldsymbol{\Psi}) \leq K$, we deduce that, if there exists such a ground state, then $E_{N}^{K+2}<\bar{E}_{N}^{K}$ as requested. The aforementioned demonstration includes an argument based upon the assumption that solutions (i.e., orbitals) to the system of PDEs

$$
\begin{aligned}
d_{k}\left(\widetilde{T}_{0}+V_{\text {en }}\right) \phi_{k}+\sum_{l m n} \tilde{c}_{k l m n} w_{l m} \phi_{k} & =\sum_{l=1}^{K} \lambda_{k l} \phi_{l}, \\
-\Delta w_{l m} & =4 \pi \phi_{l}(x) \phi_{m}(x),
\end{aligned}
$$


(here $\left.w(x)=\int_{\mathbb{R}^{3}}\left|x-x^{\prime}\right|^{-1} w\left(x^{\prime}\right) d x^{\prime}\right)$ are real-analytic functions on the region $\mathbb{R}^{3} \backslash\left\{R_{1}, \ldots, R_{L}\right\}$. This result has been established in [8] 4. Hence we conclude that if $\mathcal{J}=\mathrm{J}_{K, N}$ then $\widetilde{K}$ equals either $K$ or $K-1$.

The non-relativistic MC analogue of Proposition 9.1 was established by Lewin [29], and the quasi-relativistic HF version was shown by Enstedt and Melgaard [14]; in the latter case every "relaxed" Palais-Smale sequence is relatively compact in $\mathcal{C}_{N}$.

\section{Existence of A GROUnd State}

We address the minimization problem formulated in Definition 4.2.

Theorem 10.1. Let $K$ and $N$ be positive integers such that $K \geq N$ and $N-1<Z_{\text {tot }}<Z_{\mathrm{c}}$, where $Z_{\mathrm{tot}}=\sum_{l=1}^{L} Z_{l}$ is the total nuclear charge and $Z_{\mathrm{c}}=2 \alpha^{-1} \pi^{-1}$. If $\mathcal{J} \subset \mathcal{C}_{\mathcal{J}, K, N}$ satisfies Definition 6.1, then there exists a minimizer $(a, \varphi)$ of the quasi-relativistic $M C$ functional $\mathcal{E}_{K, N}(\cdot)$ on $\mathcal{C}_{K, N}$, i.e., a minimizer to

$$
E_{\mathcal{J}, K, N}=\inf _{\mathcal{C}_{\mathcal{J}, K, N}} \mathcal{E}_{K, N}
$$

Before proving Theorem 10.1, let us give a few explanations. To ensure that a Palais-Smale sequence converges, we need to somehow "improve" it. Since $\mathcal{E}_{K, N}$ is a $C^{2}$-functional, we may try to obtain an almost critical sequence with some information on the second derivative. This enables us to built an almost critical sequence which satisfies (9.3). Due to lack of compactness, we cannot find critical points of $\mathcal{E}_{K, N}$ and therefore we perturb the functional while, simultaneous, ensuring that the new functional has critical points of the kind, we expect for the original one. The way we shall obtain such sequences consists in applying a "perturbed variational principle" by Borwein and Preiss [5].

Proof of Theorem 10.1. First of all we note that (3.1) and the nonnegativity of $\langle\boldsymbol{E} \cdot \cdot, \cdot\rangle$ imply that $\mathcal{E}_{K, N}$ is bounded from below (uniformly) on $\mathcal{C}_{K, N}$ and we may therefore conclude existence of a minimizing sequence $\left\{\left(\tilde{a}^{(j)}, \widetilde{\boldsymbol{\Phi}}^{(j)}\right)\right\}_{j=1}^{\infty}$ to (4.6). To prove the existence of a minimizer, the idea is to apply Proposition 9.1 and, therefore, we need to verify

\footnotetext{
${ }^{4}$ The proof of this for the Hartree-Fock model is found in the first version of [8]. Later, in the second version of [8] (appearing after the publication of [2]) it was noted that the proof also works for the MC model. We acknowledge T. Ø Sørensen for informing us of this.
} 
the hypotheses therein. For this purpose we are going to use a minimization principle by Borwein and Preiss [5, Theorem 2.6] in order to obtain a new minimizing sequence. The variational space $\mathcal{C}_{K, N}$ is a complete metric space endowed with the metric

$$
d\left((a, \boldsymbol{\phi}),\left(a^{\prime}, \boldsymbol{\phi}^{\prime}\right)\right)^{2}=\left|a-a^{\prime}\right|^{2}+\left\|\boldsymbol{\phi}-\boldsymbol{\phi}^{\prime}\right\|_{\left(\mathbf{H}^{1 / 2}\left(\mathbb{R}^{3}\right)\right)^{K}}^{2}
$$

To apply the principle we introduce $Q$ as the set of functions that can be written in the following way

$$
q(a, \phi)=\frac{1}{2} \sum_{k} \alpha_{k} d\left((a, \phi),\left(b_{k}, \boldsymbol{\psi}_{k}\right)\right)^{2}, \quad(a, \phi) \in \mathcal{C}_{K, N}
$$

for some convergent sequence $\left\{\left(b_{k}, \boldsymbol{\psi}_{k}\right)\right\}_{k}$, and real, nonnegative numbers $\alpha_{k}$ satisfying $\sum_{k} \alpha_{k}=1$. Then an application of the principle provides us with a new minimization sequence $\left\{\left(a^{(j)}, \boldsymbol{\Phi}^{(j)}\right)\right\}_{j=1}^{\infty}$, such that

$$
\lim _{j \rightarrow \infty}\left\|\Phi^{(j)}-\widetilde{\boldsymbol{\Phi}}^{(j)}\right\|_{\mathbf{H}^{1 / 2}\left(\mathbb{R}^{3}\right)^{K}}=0 \quad \text { and } \lim _{j \rightarrow \infty}\left|a_{J}^{(j)}-\tilde{a}_{J}^{(j)}\right|=0
$$

and, most importantly, the variational principle asserts that $\left(a^{(j)}, \boldsymbol{\Phi}^{(j)}\right)$ minimizes

$$
\mathcal{E}_{K, N}(\cdot)+\frac{1}{j} q_{j}(\cdot)
$$

with $q_{j} \in Q$. From this we conclude that hypothesis (ii) in Assumption 6.2 is satisfied. Moreover, we can (after some straightforward computations) conclude that (9.3) holds for all $\psi$ belonging to a closed subspace $\mathbf{H}^{1 / 2}\left(\mathbb{R}^{3}\right)$ with finite codimension at most $\widetilde{K}$. This shows that Assumption 6.2(iii) holds true. Hence the existence of a minimum follows from Proposition 9.1.

\section{INFINITUDE OF NONMINIMAL SOLUTIONS}

We establish existence of infinitely many critical (or saddle) points $(a, \phi)$ for $\mathcal{E}_{K, N}$ on $\mathcal{C}_{K, N}$ by giving a new application of the Lions-FangGhoussoub approach.

Theorem 11.1. Let $K$ and $N$ be positive integers such that $K \geq N$ and $N-1<Z_{\mathrm{tot}}<Z_{\mathrm{c}}$, where $Z_{\mathrm{tot}}=\sum_{l=1}^{L} Z_{l}$ is the total nuclear charge and $Z_{\mathrm{c}}=2 \alpha^{-1} \pi^{-1}$. If $K \geq N$ and $\mathcal{J} \subset \mathcal{C}_{\mathcal{J}, K, N}$ satisfies Definition 6.1, then there exists an infinite sequence $\left\{\left(a^{(j)}, \varphi^{(j)}\right)\right\}_{j \geq 1}$ of 
distinct critical points of the $M C$ functional $\mathcal{E}_{K, N}$ on $\mathcal{C}_{\mathcal{J}, K, N}$. The functions $\varphi_{1}^{(j)}, \ldots, \varphi_{K}^{(j)}$ satisfy the quasi-relativistic $M C$ equations

$$
\begin{aligned}
\left(\left(\widetilde{T}_{0}+V_{\mathrm{en}}\right) \boldsymbol{D}^{(j)}+2 \boldsymbol{E}_{\boldsymbol{\varphi}^{(j)}}\right) \boldsymbol{\varphi}^{(j)}+\Lambda^{(j)} \cdot \boldsymbol{\varphi}^{(j)} & =0 \\
T_{\boldsymbol{\varphi}^{(j)}} \cdot a & =\zeta \cdot a^{(j)}
\end{aligned}
$$

for some $\Lambda^{(j)} \geq 0$. Moreover, as $j \rightarrow \infty$, the corresponding sequence of wave functions $\left\{\boldsymbol{\Psi}^{(j)}\right\}_{j \geq 1}$ satisfies

$$
\begin{array}{r}
\mathcal{E}\left(\boldsymbol{\Psi}^{(j)}\right)<0, \quad \mathcal{E}\left(\boldsymbol{\Psi}^{(j)}\right) \rightarrow 0, \quad \Lambda^{(j)} \rightarrow 0 \\
\sqrt{\boldsymbol{D}^{(j)}} \widetilde{T}_{0}^{1 / 2} \boldsymbol{I}_{K} \boldsymbol{\varphi}^{(j)} \longrightarrow 0 \quad \text { strongly in }\left(L^{2}\left(\mathbb{R}^{3}\right)\right)^{K}
\end{array}
$$

To prove that there exists a critical point at infinitely many distinct levels, we first apply an abstract result on critical points by Fang and Ghoussoub [15]. Consider the $C^{2}$-functional $\mathcal{E}_{K, N}$ on the complete, analytic Hilbert-Riemann manifold $\mathcal{C}_{K, N}$; see Theorem 4.1. (In prior applications of the Lions-Fang-Ghoussoub approach the necessary geometric properties of the variational space, imposed in [15, Theorem 1.7], were not mentioned [31, 15, 29].)

We note that $\mathbb{Z}_{2}=\{-1,1\}$ equipped with multiplication as binary operation and the discrete topology can be considered as a compact (0-dimensional) Lie group, with group action on $\mathbb{S}^{k-1}$ of $\mathbb{R}^{d}$ given by

$$
( \pm, x) \mapsto \pm x
$$

and, on $\mathcal{C}_{K, N}$ we introduce the group action

$$
(-) \cdot{ }_{\phi}(a, \phi)=(a,-\phi),
$$

Then $\mathcal{E}_{K, N}$ is $\left(\mathbb{Z}_{2}\right)_{\phi^{-}}$invariant ${ }^{5}$ on $C_{K, N}$, i.e., $\mathcal{E}_{K, N}(a, \phi)=\mathcal{E}_{K, N}(a,-\boldsymbol{\phi})$.

\footnotetext{
${ }^{5}$ The significance of this choice of action is as follows [29]: Let $\Theta_{k}$ be the family of all odd continuous maps from $\mathbb{S}^{k-1}$ into the sphere of the $N$-particle space $\mathbf{H}_{\mathrm{a}}^{1 / 2}\left(\mathbb{R}^{3 N}\right)$ and let $\Pi_{K, N}$ be the natural projection from $\mathcal{C}_{K, N}$ into $\mathbf{H}_{\mathrm{a}}^{1 / 2}\left(\mathbb{R}^{3 N}\right)$, i.e.,

$$
\Pi_{K, N}:(a, \phi) \mapsto \quad \Psi=\sum_{J} a_{J} \phi_{J}
$$

Then the eigenvalues of $T_{N, \boldsymbol{Z}, \alpha}$ can be expressed by

$$
\nu_{k}=\min _{g \in \Theta_{k}} \max _{\Psi \in g\left(\mathbb{S}^{k-1}\right)} \mathcal{E}(\Psi)
$$

The choice (11.4) ensures that $g=\Pi_{K, N} \circ h$ belongs to $\Theta_{k}$ for all $\left(\mathbb{Z}_{2}\right)_{\phi}$-equivariant functions $h: \mathbb{S}^{k-1} \rightarrow \mathcal{C}_{K, N}$ (in other words, $\Pi_{K, N} \circ h$ is odd).
} 
Next we make preparations for the min-max principle: For each $k \in \mathbb{N}$, we consider the following homotopic class of order $k$

$$
\mathrm{H}_{k}^{\phi}=\left\{\mathrm{M}: \mathrm{M}=h\left(\mathbb{S}^{k-1}\right) \text { for some odd } h \in C_{\left(\mathbb{Z}_{2}\right)}\left(\mathbb{S}^{k-1} ; \mathcal{C}_{K, N}\right)\right\}
$$

where $\mathbb{S}^{k-1}$ is the unit sphere in the Euclidean space $\mathbb{R}^{k}$. For the full MC method we define

$$
l_{k}^{K}=\inf _{\mathbf{M}_{\in} \mathrm{H}_{k}^{\phi}} \max _{(a, \phi) \in \mathrm{M}} \mathcal{E}_{K, N}(a, \phi),
$$

and, analogously, for the partial MC method we can define $l_{k}^{\mathcal{J}, K}$ by replacing $\mathcal{C}_{N, K}$ by $\mathcal{C}_{\mathcal{J}, K, N}$ in (11.5)-(11.6). The choice of the min-max in (11.6) is similar to the one in [31, 19, 35, 29].

Proof of Theorem 11.1. Since the min-max level (11.6) is defined via $\left(\mathbb{Z}_{2}\right)_{\phi}$-homotopic classes of dimension $k$ for each $k$, an application of the abstract results by Fang and Ghoussoub [15, Theorem 1.7] enables us to extract a sequence $\left\{\left(a^{(j)}, \phi^{(j)}\right)\right\}$ (one sequence for each $k$ ) satisfying Assumption 6.2 with $k=m$. In view of Proposition 9.1, this sequence converges (up to extracting subsequences) to some critical point $\left(a^{(k)}, \varphi^{(k)}\right)$ of $\mathcal{E}_{\widetilde{K}, N}$ on $\mathcal{C}_{\mathcal{J}, \widetilde{K}, N}$ with $\widetilde{K} \leq K$. By adding any $\left(\varphi_{\widetilde{K}+1}^{(k)}, \ldots, \varphi_{K}^{(k)}\right)$ we obtain solutions of (11.1)-(11.2) with $\Lambda \geq 0$. We proceed to the study of $\left\{l_{k}^{\mathcal{J}, K}\right\}$. Both $K \geq N$ and $\mathcal{J}$ are fixed and, therefore, we suppress these superscripts in the sequel.

We claim that $-\infty<l_{k} \leq l_{k+1}<0$ for each $k \in \mathbb{N}$ and that $l_{k} \rightarrow 0$ (as $k \rightarrow \infty$ ). The monotonicity of $\left\{l_{k}\right\}_{k=1}^{\infty}$ is a direct consequence of how we have defined $\mathrm{H}_{k}^{\phi}$ and since $\mathcal{E}_{K, N}$ is uniformly bounded below on $\mathcal{C}_{K, N}$, we immediately get that $l_{k}>-\infty$. If we introduce $j_{k}=k \cdot K$, then an application of Lemma 8.1 ensures that, for each $k \geq 1$, there exists a $j_{k}$-dimensional subspace $\mathcal{H}_{k}$ of $\mathbf{H}^{1 / 2}\left(\mathbb{R}^{3}\right)$ such that for all $\phi \in$ $\mathcal{H}_{k}$ with $\|\phi\|_{L^{2}\left(\mathbb{R}^{3}\right)}=1$ (we denote the unit sphere in this subspace by $\left.\widetilde{\mathbb{S}}^{k-1}\right)$, one has

$$
\tilde{\mathfrak{t}}_{0}[\phi, \phi]+\mathfrak{v}_{\mathrm{e}}[\phi, \phi]+\int_{\mathbb{R}^{3}} \int_{\mathbb{R}^{3}} \frac{\rho(x)\left|\phi\left(x^{\prime}\right)\right|^{2}}{\left|x-x^{\prime}\right|} \mathrm{d} x \mathrm{~d} x^{\prime} \leq-\epsilon_{k},
$$

for some $\epsilon_{k}>0$. We can always build an $L^{2}$-orthonormal basis $\left\{\phi_{n}\right\}_{n=1}^{j_{k}}$ of $\mathcal{H}_{k}$ consisting of radially symmetric $C_{0}^{\infty}\left(\mathbb{R}^{3}\right)$-functions satisfying

$$
\operatorname{supp} \phi_{m} \cap \operatorname{supp} \phi_{n}=\emptyset, \quad m \neq n
$$


Without loss of generality, we assume that $\{1, \ldots, N\} \in \mathcal{J}$. We may re-write the quasi-relativistic (QR) Hartree-Fock functional $\mathcal{E}^{\mathrm{QR}}$ as

$$
\begin{aligned}
\mathcal{E}^{\mathrm{QR}}\left(\phi_{1}, \ldots, \phi_{N}\right)= & \sum_{n=1}^{N}\left(\tilde{\mathfrak{t}}_{0}\left[\phi_{n}, \phi_{n}\right]+\mathfrak{v}_{\mathrm{en}}\left[\phi_{n}, \phi_{n}\right]+\mathfrak{v}_{\mu_{n} *(1 /|x|)}\left[\phi_{n}, \phi_{n}\right]\right) \\
& -\frac{1}{2} \sum_{m \neq n=1}^{N} \int_{\mathbb{R}^{3}}\left(\left(\phi_{m} \phi_{n}\right) * \frac{1}{|x|}\right) \phi_{m} \phi_{n}
\end{aligned}
$$

with

$$
\mu_{m}=\sum_{n=1, n \neq m}^{N}\left|\phi_{n}\right|^{2} \text { and } \int_{\mathbb{R}^{3}} \mu_{m}=N-1 .
$$

The nonnegativity of all terms

$$
\int_{\mathbb{R}^{3}}\left(\left(\phi_{m} \phi_{n}\right) * \frac{1}{|x|}\right) \phi_{m} \phi_{n}, \quad m \neq n
$$

implies that, for any continuous $\left(\mathbb{Z}_{2}\right)_{\phi}$-function $h: \mathbb{S}^{k-1} \rightarrow \mathcal{C}_{\mathcal{J}, K, N}$ defined by

$$
\left(b_{1}, \ldots, b_{k}\right) \mapsto\left(\left(\begin{array}{c}
1 \\
0 \\
\vdots \\
0
\end{array}\right),\left(\begin{array}{c}
b_{1} \phi_{1}+\cdots+b_{k} \phi_{k} \\
\vdots \\
\vdots \\
b_{1} \phi_{k(K-1)}+\cdots+b_{k} \phi_{k K}
\end{array}\right)\right)
$$

we have that, using (11.7),

$$
\begin{aligned}
\mathcal{E}_{K, N} & \left(h\left(b_{1}, \ldots, b_{k}\right)\right)= \\
= & \mathcal{E}_{N}\left(\left(b_{1} \phi_{1}+\cdots+b_{k} \phi_{k}\right) \wedge \cdots \wedge\left(b_{1} \phi_{k(N-1)+1}+\cdots+b_{k} \phi_{K k}\right)\right) \\
= & \mathcal{E}_{N}\left(\sum_{n_{1}=1}^{k} \cdots \sum_{n_{N}=(N-1) k+1}^{k N} b_{n_{1}} \cdots b_{n_{N}-(N-1) k}\left(\phi_{n_{1}} \wedge \cdots \wedge \phi_{n_{N}}\right)\right) \\
= & \sum_{n_{1}=1}^{k} \cdots \sum_{n_{N}=(N-1) k+1}^{k N}\left(b_{n_{1}} \cdots b_{n_{N}-(N-1) k}\right)^{2} \mathcal{E}_{N}\left(\phi_{n_{1}} \wedge \cdots \wedge \phi_{n_{N}}\right) \\
= & \sum_{n_{1}=1}^{k} \cdots \sum_{n_{N}=(N-1) k+1}^{k N}\left(b_{n_{1}} \cdots b_{n_{N}-(N-1) k}\right)^{2} \mathcal{E}^{\mathrm{QR}}\left(\phi_{n_{1}}, \ldots, \phi_{n_{N}}\right) \\
\leq & \sum_{n_{1}=1}^{k} \cdots \sum_{n_{N}=1}^{k}\left(b_{n_{1}} \cdots b_{n_{N}}\right)^{2}\left(-N \epsilon_{k}\right)=-N \epsilon_{k}<0
\end{aligned}
$$


for all $\left(b_{1}, \ldots, b_{k}\right) \in \mathbb{S}^{k-1}$. This proves $l_{k} \leq \max _{h\left(\mathbb{S}^{k-1}\right)} \mathcal{E}_{K, N}(h)<0$. To prove that $\lim _{k \rightarrow \infty} l_{k}=0$, we use the separability of $\left(\mathbf{H}^{1 / 2}\left(\mathbb{R}^{3}\right)\right)^{K}$ by considering a nested sequence of finite-dimensional subspaces $\mathcal{W}_{k}$ of $\left(\mathbf{H}^{1 / 2}\left(\mathbb{R}^{3}\right)\right)^{K}$ such that $\operatorname{dim}\left(\mathcal{W}_{k}\right)=k$ and $\cup_{k} \mathcal{W}_{k}$ is dense in $\left(\mathbf{H}^{1 / 2}\left(\mathbb{R}^{3}\right)\right)^{K}$. Define $\mathcal{V}_{k}$ as the orthogonal complement of $\mathcal{W}_{k-1}$. From above, we know that $l_{k}<0$ for every $k \geq 1$ and, therefore, we can find $\mathrm{M}_{k}^{\phi} \in \mathrm{H}_{k}^{\phi}$ such that

$$
l_{k} \leq \max _{\mathrm{M}_{k}^{\phi}} \mathcal{E}_{K, N}<\frac{l_{k}}{2}
$$

Suppressing the supscripts for simplicity, we may assume that $\mathrm{M}_{k} \cap \mathcal{V}_{k}=$ $\emptyset$, let $\pi_{k}$ be the orthogonal projection from $\mathbf{H}^{1 / 2}\left(\mathbb{R}^{3}\right)$ onto $\mathcal{W}_{k}$. Then (note that $\mathcal{V}_{k+1}=\operatorname{Ker}\left(\pi_{k}\right) \subset \mathcal{V}_{k}$ )

$$
\pi_{k-1}\left(\mathrm{M}_{k}\right) \subset \mathcal{W}_{k-1} \backslash\{0\} \cong \mathbb{R}^{k-1} \backslash\{0\} .
$$

Since $\mathrm{M}_{k}^{\phi}=h\left(\mathbb{S}^{k-1}\right)$, for some $h \in C_{\left(\mathbb{Z}_{2)} \phi\right.}\left(\mathbb{S}^{k-1} ; \mathcal{C}_{K, N}\right)$ we have the existence of a continuous and odd map from $\mathbb{S}^{k-1}$ to $\mathbb{R}^{k-1} \backslash\{0\}$. By following Rabinowitz [35] and using the Borsuk-Ulam theorem we will now get the existence of two antipodal points on $\mathbb{S}^{k-1}$ which maps (due to symmetry) to 0 and hence we have reached a contradiction. For each $k$ fix some $\varphi^{(k)} \in \mathrm{M}_{k} \cap \mathcal{V}_{k}$ and extract a $\mathbf{H}^{1 / 2}\left(\mathbb{R}^{3}\right)^{K}$-weakly convergent subsequence $\left\{\boldsymbol{\varphi}^{(k)}\right\}_{k}$. Hence we have $\left\{a^{(k)}, \boldsymbol{\varphi}^{(k)}\right\}$ such that $a^{(k)}$ is bounded and $\boldsymbol{\varphi}^{(k)} \rightarrow 0$ weakly in $\mathbf{H}^{1 / 2}\left(\mathbb{R}^{3}\right)^{K}$. Then an application of Lemma 5.1 yields

$$
\liminf _{k \rightarrow \infty} \mathcal{E}_{K, N}\left(a^{(k)}, \varphi^{(k)}\right)=0 .
$$

The latter, together with (11.8) implies that $\lim _{k \rightarrow \infty} l_{k}=0$.

Let us now prove the properties of the sequence $\left\{a^{(k)}, \boldsymbol{\varphi}^{(k)}\right\}_{k \geq 1}$ of distinct solutions. We have already seen that we may assume that $-\infty<l_{k}<l_{k+1}<0$ so we may find a sequence such that

$$
-\infty<l_{k-1}<l_{k}=\mathcal{E}_{K, N}\left(a^{(k)} \varphi^{(k)}\right)<l_{k+1}<0 .
$$

We conclude that

$$
\mathcal{E}_{K, N}\left(a^{(k)}, \varphi^{(k)}\right) \rightarrow 0 .
$$

For every $k \geq 1$ there exists $\left(a^{(k)}, \varphi^{(k)}\right)$ and a nonnegative matrix $\Lambda^{(k)}$ such that $\mathcal{E}_{K, N}\left(a^{(k)}, \boldsymbol{\varphi}^{(k)}\right)=l_{k}$ and $\left(a^{(k)}, \boldsymbol{\varphi}^{(k)}\right)$ is a solution to the MC equations (11.1)-(11.2). We infer that, as $k \rightarrow \infty$,

$$
\left\langle\boldsymbol{E}_{\boldsymbol{\varphi}^{(k)}} \boldsymbol{\varphi}^{(k)}, \boldsymbol{\varphi}^{(k)}\right\rangle+\operatorname{Tr}\left[\Lambda^{(k)}\right]=-\mathcal{E}_{K, N}\left(a^{(k)}, \boldsymbol{\varphi}^{(k)}\right)=-l_{k} \longrightarrow 0
$$

and, consequently, as $k \rightarrow \infty$,

$$
\left\langle\boldsymbol{E}_{\boldsymbol{\varphi}^{(k)}} \boldsymbol{\varphi}^{(k)}, \boldsymbol{\varphi}^{(k)}\right\rangle \rightarrow 0 \text { and } \Lambda^{(k)} \rightarrow 0
$$


In view of (9.1), we see that the sequence $\left\{\sqrt{\boldsymbol{D}^{(k)}} \boldsymbol{\varphi}^{(k)}\right\}$ is bounded in $\mathbf{H}^{1 / 2}\left(\mathbb{R}^{3}\right)^{K}$. Hence we may suppose that $a_{J}^{(k)} \varphi_{n}^{(k)}$ converges weakly to some $\varphi_{J, n}$ in $\mathbf{H}^{1 / 2}\left(\mathbb{R}^{3}\right)^{K}$, strongly to $\varphi_{J, n}$ in $L_{\mathrm{loc}}^{2}\left(\mathbb{R}^{3}\right)$ and a.e. for all $n=1, \ldots, K$ and all $J$ satisfying $n \in J$. From (11.11) we deduce that $\varphi_{J, n}=0$ and, therefore, in the sense of forms,

$$
\left\langle\left(\widetilde{T}_{0}+V_{\text {en }}\right) \boldsymbol{D}^{(k)} \boldsymbol{\varphi}^{(k)}, \boldsymbol{\varphi}^{(k)}\right\rangle \longrightarrow 0
$$

The left-hand side of (11.12) has the decreasing property (5.2) and, therefore, we conclude that $\sqrt{\boldsymbol{D}^{(k)}} \widetilde{T}_{0}^{1 / 2} \boldsymbol{I}_{K} \boldsymbol{\varphi}^{(k)} \rightarrow 0$ strongly in $L^{2}\left(\mathbb{R}^{3}\right)^{K}$.

\section{Comparison With FUll QUANTUM MEChanical QUANTITIES}

We compare the eigenvalues of the full quantum mechanical Hamiltonian $T_{N, \boldsymbol{Z}, \alpha}$, i.e.,

$$
\nu_{k}=\min _{\operatorname{dim} V=k} \max _{\substack{\boldsymbol{\Psi} \in V,\|\boldsymbol{\Psi}\|=1}} \mathcal{E}(\boldsymbol{\Psi})
$$

with certain critical points of $\mathcal{E}_{K, N}$ on $\mathcal{C}_{K, N}$. For this purpose we introduce the following group action of $\mathbb{Z}_{2}$ on $\mathcal{C}_{K, N}$, in addition to the one already mentioned in (11.4),

$$
(-) \cdot{ }_{a}(a, \boldsymbol{\Phi})=(-a, \boldsymbol{\Phi})
$$

We say that a function $f: \mathbb{S}^{k-1} \rightarrow \mathcal{C}_{K, N}$ is $\left(\mathbb{Z}_{2}\right)_{a}$-equivariant provided

$$
h(x)=(a, \mathbf{\Phi}) \quad \text { implies that } \quad h(-x)=(-a, \mathbf{\Phi}) .
$$

It is apparent that $\mathcal{E}_{K, N}$ is $\left(\mathbb{Z}_{2}\right)_{a}$-equivariant on $\mathcal{C}_{K, N}$, i,e,

$$
\mathcal{E}_{K, N}(a, \boldsymbol{\Psi})=\mathcal{E}_{K, N}(-a, \boldsymbol{\Phi}) .
$$

For $1 \leq k \leq\left(\begin{array}{l}K \\ N\end{array}\right)$, we define

$$
\nu_{k}^{K}=\inf _{\left.h \in C_{\left(\mathbb{Z}_{2}\right)_{c}} \mathbb{S}^{k-1}, \mathcal{C}_{K, N}\right)} \max _{(a, \mathbf{\Phi}) \in h\left(\mathbb{S}^{k-1}\right)} \mathcal{E}_{K, N}(a, \boldsymbol{\Phi})
$$

The following quasi-relativistic result is similar to the non-relativistic result by Lewin [29, Theorem 2 (ii)]. Its proof is also similar and, therefore, we omit it.

Theorem 12.1. Let $K$ and $N$ be positive integers such that $K \geq N$ and $N-1<Z_{\text {tot }}<Z_{\mathrm{c}}$, where $Z_{\mathrm{tot}}=\sum_{l=1}^{L} Z_{l}$ is the total nuclear charge and $Z_{\mathrm{c}}=2 \alpha^{-1} \pi^{-1}$. Suppose $1 \leq k \leq\left(\begin{array}{l}K \\ N\end{array}\right)$. Then there exists $\left(a^{(k)}, \boldsymbol{\varphi}^{(k)}\right)$ belonging to $\mathcal{C}_{K, N}$ such that $\mathcal{E}_{K, N}\left(a^{(k)}, \varphi^{(k)}\right)=\nu_{k}^{K}$. Furthermore, $\nu_{k}^{K}$ fulfills

$\nu_{k} \leq \nu_{k}^{K} \leq \lambda_{k}^{K}$

and, in particular, $\lim _{K \rightarrow \infty} \nu_{k}^{K}=\nu_{k}$. 
Acknowledgement. The authors acknowledge M. Lewin [30] for pointing out errors in the original version of [2]. This revised version of [2] entirely avoids the use of density operator formalism.

\section{REFERENCES}

[1] Sh. Agmon, Lectures on elliptic boundary value problems. Nostrand Mathematical Studies, No. 2 D. Van Nostrand Co., Inc., Princeton, N.J.-Toronto-London, 1965.

[2] C. Argaez, M. Melgaard, Solutions to quasi-relativistic multi-configurative Hartree-Fock equations in quantum chemistry, Nonlinear Analysis: theory, methods, and applications $\mathbf{7 5}$ (2012), 384-404.

[3] T. Bartsch, Z.-Q.-Wang, M. Willem, The Dirichlet problem for superlinear elliptic equations, Stationary Partial Differential Equations Vol 2, 1-55, Handb. Diff. Eqn., Elsevier/North-Holland, Amsterdam, 2005.

[4] J.-M. Barbaroux, W. Farkas, B. Helffer, H. Siedentop, On the Hartree-Fock equations of the electron-positron field, Comm. Math. Phys 255 (2005), no. 1, 131-159.

[5] J. Borwein, D. Preiss, A smooth variational principle with applications to subdifferentiability and to differentiability of convex functions. Trans. A.M.S. 303 (1987), 517-527.

[6] E. Chiumiento, M. Melgaard, Stiefel and Grassmann manifolds in Quantum Chemistry (2011), submitted elsewhere.

[7] S. Cingolani, M. Lazzo, Multiple semiclassical standing waves for a class of nonlinear Schrödinger equations, Topol. Methods Nonlin. Anal. 10 (1997), no. $1,1-13$.

[8] Dall'Acqua, S. Fournais, T. Ø. Sørensen, E. Stockmeyer, Real analyticity away from the nucleus of pseudorelativistic Hartree-Fock orbitals, Analysis \& PDE, to appear (2011).

[9] A. Dall'Acqua, T. Ø. Sørensen, E. Stockmeyer, Hartree-Fock theory for pseudorelativistic atoms. Ann. Henri Poincaré 9 (2008), no. 4, 711-742.

[10] E. N. Dancer, Finite Morse index solutions of exponential problems, Ann. Inst. H. Poincaré Anal. Non Linéaire 25 (2008), 173-179.

[11] D. G. de Figueiredo, P. N. Srikanth, S. Santra, Non-radially symmetric solutions for a superlinear Ambrosetti-Prodi type problem in a ball. Commun. Contemp. Math. 7 (2005), no. 6, 849-866.

[12] D. E. Edmunds, W. D. Evans, Spectral Theory and Differential Operators. Clarendon Press, Oxford University Press, New York, 1987.

[13] M. Enstedt, M. Melgaard, Existence of a solution to Hartree-Fock equations with decreasing magnetic fields. Nonlinear Anal. 69 (2008), no. 7, 2125-2141.

[14] M. Enstedt, M. Melgaard, Existence of infinitely many solutions to the quasirelativistic Hartree-Fock equations, Int. J. Math. Math. Sci. Volume 2009, Article ID 651871, 20 pages.

[15] G. Fang, N. Ghoussoub, Morse-type information on Palais-Smale sequences obtained by min-max principles, Comm. Pure Appl. Math 47 (1994), no 12, 1595-1653. 
[16] G. Flores, P. Padilla, Y. Tonegawa, Higher energy solutions in the theory of phase transitions: a variational approach, J. Diff. Eqn. 169 (2001), no. 1, 190-207.

[17] G. Friesecke, The multiconfiguration equations for atoms and molecules: charge quantization and existence of solutions. Arch. Rational Mech. Anal. 169 (2003), 35-71.

[18] G. Friesecke, On the infinitude of non-zero eigenvalues of the single-electron density matrix for atoms and molecules. Proc. R. Soc. Lond. A 459 (2003), $47-52$.

[19] N. Ghoussoub, Duality and Perturbation Methods in Critical Point Theory, Cambridge University Press, 1993.

[20] N. Ghoussoub, C. Yuan, Multiple Solutions for quasi-linear PDEs involving the critical Sobolev and Hardy exponents, Trans. Amer. Math. Soc. 352 (2000), no $12,5703-5743$.

[21] I. W. Herbst, Spectral theory of the operator $\left(p^{2}+m^{2}\right)^{1 / 2}-Z e^{2} / r$. Comm. Math. Phys. 53 (1977), no. 3, 285-294.

[22] L. Jeanjean, On the existence of bounded Palais-Smale sequences and application to a Landesman-Lazer type problem set on $\mathbb{R}^{N}$, Proc. Roy. Soc. Edinburgh, 129A (1999), 787-809.

[23] F. Jensen, Introduction to Computational Chemistry, 2nd edition, Wiley, 2006.

[24] T. Kato, Perturbation Theory for Linear Operators. Reprint of the 1980 edition. Classics in Mathematics. Springer-Verlag, Berlin, 1995.

[25] A. C. Lazer, S. Solimini, Nontrivial solutions of operator equations and Morse indices of critical points of min-max type. Nonlinear Anal. 12 (1988), no. 8, $761-775$.

[26] R. T. Lewis, H. Siedentop, S. Vugalter, The essential spectrum of relativistic multi-particle operators, Ann. Inst. Henri Poincaré 67 (1997), no. 1, 1-28.

[27] C. Le Bris, A general approach for multiconfiguration methods in quantum molecular chemistry. Ann. Inst. Henri Poincaré 11 (1994), 441484.

[28] I. N. Levine, Quantum Chemistry, Prentice Hall, 1991.

[29] M. Lewin, Solutions to the multiconfiguration equations in quantum chemistry, Arch Rational Mech. Anal. 171 (2004), 83-114.

[30] M. Lewin, private communication, November 22, 2011.

[31] P.-L. Lions, Solutions of Hartree-Fock equations for Coulomb systems. Comm. Math. Phys. 109 (1987), no. 1, 33-97.

[32] P.-O. Löwdin, Quantum Theory of Many-Particle systems I. Physical Interpretations by Means of Density Matrices, Natural Spin-Orbitals, and Convergence Problems in the Method of Configurational Interaction. Phys. Rev. 97 (1955), 14741489 .

[33] E. A. Mazepa, On the existence of entire solutions of a semilinear elliptic equation on noncompact Riemannian manifolds. (Russian) Mat. Zametki $\mathbf{8 1}$ (2007), no. 1, 153-156; translation in Math. Notes 81 (2007), no. 1-2, 135-139.

[34] R. G. Parr, W. Yang, Density-functional theory of atoms and molecules, Oxford University Press, 1989.

[35] P. H. Rabinowitz, Variational methods for nonlinear eigenvalue problems. Eigenvalues of non-linear problems (Centro Internaz. Mat. Estivo (C.I.M.E.), III Ciclo, Varenna, 1974), pp. 139-195. Edizioni Cremonese, Rome, 1974. 
[36] G. Rozenblum, M. Melgaard, Schrödinger operators with singular potentials, in: Stationary partial differential equations. Vol. II, 407-517, Handb. Differ. Equ., Elsevier/North-Holland, Amsterdam, 2005.

[37] J. P. Solovej, Proof of the ionization conjecture in a reduced Hartree-Fock model. Invent. Math. 104 (1991), no. 2, 291-311.

[38] A. Szabo, N. S. Ostlund, Modern quantum chemistry: an introduction to advanced electronic structure theory, MacMillan, 1982.

[39] K. Tanaka, Morse indices at critical points related to the symmetric mountain pass theorem and applications, Comm. in part. diff. equations 14 (1989), no. $1,99-128$.

[40] K. Tanaka, Periodic solutions for singular Hamiltonian systems and closed geodesics on noncompact Riemannian manifolds, Ann. Inst. H. Poincaré Anal. Non Linéaire 17 (2000), no. 1, 1-33.

[41] G. M. Zhislin, S. A. Vugalter, On the discrete spectrum of Hamiltonians for pseudorelativistic electrons, Izv. Math. 66 (2002), no. 1, 71102

(C. Argaez) School of Mathematical Sciences, Dublin Institute of Technology, Dublin 8, Republic of Ireland

(M. Melgaard) School of Mathematical Sciences, Dublin Institute of Technology, Dublin 8, Republic of Ireland

E-mail address: mmelgaard@dit.ie 